\title{
On $p$-Adic Absolute CM-Periods II
}

\author{
By
}

\author{
Tomokazu KASHIO* and Hiroyuki YosHIDA**
}

\begin{abstract}
In part I of this paper, we studied the $p$-adic absolute CM-period symbol in the completely split case. We presented a conjecture which predicts the exact value of this symbol with supporting evidences. In this part II, we study the properties of this symbol in the general case and clarify its relation to $p$-adic periods.
\end{abstract}

\section{Introduction}

Let $F$ be a totally real algebraic number field and $K$ be a CM-field which is abelian over $F$. We fix an embedding $F \subset \mathbf{C}_{p}$ and let $\mathfrak{p}$ be the prime ideal of $F$ induced from this embedding. For $\tau \in \operatorname{Gal}(K / F)$, the $p$-adic absolute CM-period symbol is defined by the formula

$$
l g_{p, K / F}(\mathrm{id}, \tau)=-\frac{\mu(\tau)}{2 h_{0}} \log _{p} \alpha_{0}+\frac{1}{|G|} \sum_{\chi \in \hat{G}_{-}} \frac{\chi(\tau)}{L(0, \chi)} \sum_{c \in \mathfrak{C}_{\mathfrak{f}(\chi) \mathfrak{p}}} \chi(c) X_{p}(c)
$$

Here we put $\mathfrak{p}^{h_{0}}=\left(\alpha_{0}\right)$ with a totally positive $\alpha_{0}, h_{0}$ being the class number of $F$ in the narrow sense; $\mu(\tau)=1$ if $\tau=\mathrm{id}, \mu(\tau)=-1$ if $\tau=\rho$ and $\mu(\tau)=$ 0 otherwise, where $\rho$ denotes the complex conjugation; $G=\operatorname{Gal}(K / F)$ and $\widehat{G}_{-}$denotes the set of all odd characters of $G ; \mathfrak{f}(\chi)$ denotes the finite part of the conductor of $\chi ; \mathfrak{C}_{\mathfrak{f}(\chi) \mathfrak{p}}$ denotes the ideal class group of $F$ modulo $\mathfrak{f}(\chi) \mathfrak{p}$ times the product of all archimedean primes; $X_{p}(c)$ is a certain class invariant which is constructed using the division values of the $p$-adic logarithmic multiple gamma functions. For details we refer the reader to $\S 4$ of part I. When $\mathfrak{p}$

Communicated by A. Tamagawa. Received June 28, 2007. Revised September 4, 2008, October 15, 2008.

2000 Mathematics Subject Classification(s): 11G15, 11G99, 11S80, 14F30.

*Department of Mathematics, Kyoto University, Kyoto 606-8502, Japan.

**Department of Mathematics, Kyoto University, Kyoto 606-8502, Japan.

(C) 2009 Research Institute for Mathematical Sciences, Kyoto University. All rights reserved. 
splits completely in $K$, Conjecture A of part I gives an explicit prediction on $l g_{p, K / F}(\mathrm{id}, \tau)$ as follows. We extend the embedding $F \subset \mathbf{C}_{p}$ to an embedding $K \subset \mathbf{C}_{p}$ and let $\mathfrak{P}$ be the prime ideal of $K$ lying above $\mathfrak{p}$ induced from this embedding. Put $\mathfrak{P}^{h_{K}}=(\alpha)$ where $h_{K}$ is the class number of $K$. Then the conjecture states that

$$
l g_{p, K}(\mathrm{id}, \tau)=\frac{1}{2 h_{K}} \log _{p}\left(\alpha^{\tau^{-1} \rho} / \alpha^{\tau^{-1}}\right)+\sum_{i=1}^{n-1} a_{i} \log _{p} \epsilon_{i}
$$

with $a_{i} \in \mathbf{Q}$. Here $n=[F: \mathbf{Q}]$ and $\epsilon_{1}, \ldots, \epsilon_{n-1}$ are generators of the group of all totally positive units of $F$. The "correction term" $\sum_{i=1}^{n-1} a_{i} \log _{p} \epsilon_{i}$ was also predicted explicitly. The purpose of this part II is to clarify the nature of the $p$-adic absolute CM-period symbol in the general case.

Let $M$ be a motive over $\mathbf{Q}$. In the complex case, the periods (Deligne's periods [D]) of $M$ are defined using the comparison isomorphism

$$
I^{ \pm}: H_{B}^{ \pm}(M) \otimes_{\mathbf{Q}} \mathbf{C} \cong H_{\mathrm{DR}}^{ \pm}(M) \otimes_{\mathbf{Q}} \mathbf{C}
$$

in the usual notation. Shimura's period symbol can be interpreted in terms of motives (cf. [B11]). In the $p$-adic case, the period is defined by the comparison isomorphism

$$
I_{D R}: H_{p}(M) \otimes_{\mathbf{Q}_{p}} B_{\mathrm{DR}} \cong H_{\mathrm{DR}}(M) \otimes_{\mathbf{Q}} B_{\mathrm{DR}} .
$$

(For the notation, see the text.) We will clarify the relation of our symbol to the $p$-adic period in this sense.

In $\S 1 \sim \S 3$, we study the $p$-adic period of the motive attached to an algebraic Hecke character of a CM-field. In $\S 4$, we introduce a certain period invariant $Q$ which is constructed by the action of the Frobenius map on the $p$-adic period. In $\S 5$, we state our main conjecture which relates $l g_{p, K}(\mathrm{id}, \tau)$ to a $Q$-invariant. In $\S 6 \sim \S 7$, we investigate how far we can recover the $p$-adic period itself from the $Q$-invariant. In $\S 8$, we present some more supporting evidences for our main conjecture.

This paper has three appendices in which we give proofs of relevant results as promised in part I.

The authors would like to thank Professor Don Blasius for useful discussions. The authors would like to thank the referee for his careful reading of the manuscript and useful suggestions.

Notation. For a prime number $p, \overline{\mathbf{Q}_{p}}$ denotes an algebraic closure of $\mathbf{Q}_{p}$; $\mathbf{C}_{p}$ denotes the completion of $\overline{\mathbf{Q}_{p}}$. We fix an algebraic closure $\overline{\mathbf{Q}}$ of $\mathbf{Q}$ in $\mathbf{C}$. By 
an algebraic number field, we understand an algebraic extension of $\mathbf{Q}$ of finite degree contained in $\overline{\mathbf{Q}}$. We denote by $\rho$ the complex conjugation.

Let $F$ be an algebraic number field. The ring of integers and the group of units of $F$ are denoted by $\mathcal{O}_{F}$ and $E_{F}$ respectively. We denote by $E_{F}^{+}$the group of all totally positive units of $F$. The class number of $F$ is denoted by $h_{F}$. We denote by $J_{F}$ (resp. $J_{p, F}$ ) the set of all isomorphisms of $F$ into $\mathbf{C}$ (resp. $\mathbf{C}_{p}$ ). For a prime ideal $\mathfrak{p}$ of $F, F_{\mathfrak{p}}$ denotes the completion of $F$ at $\mathfrak{p}$. If $F$ is a totally real algebraic number field of degree $n, \infty_{1}, \ldots, \infty_{n}$ denote all archimedean primes of $F$. For an integral ideal $\mathfrak{f}$ of $F, \mathfrak{C}_{\mathfrak{f}}$ denotes the ideal class group modulo $\mathfrak{f} \infty_{1} \cdots \infty_{n}$. For $a \in F, a \gg 0$ means that $a$ is totally positive. By a CM-field, we understand a totally imaginary quadratic extension of a totally real algebraic number field. Throughout the paper, except in $\S 8$, we exclusively use the left action of Galois groups.

We refer the formulas and statements of Part I by prefixing I to the numbers and labels of them. For example, (I.1.6) means the formula (1.6) of Part I, Conjecture I.A means Conjecture A of Part I, etc.

\section{$\S 1$. Basic Comparison Isomorphisms}

The comparison isomorphisms recalled in this section were conjectured by Fontaine ([Fo1]). They were established by the work of many mathematicians. See Faltings $[\mathrm{Fa}]$, Tsuji $[\mathrm{T}]$ for example. The latter paper gives a very nice exposition on the topic.

Let $K$ be a finite extension of $\mathbf{Q}_{p}$ contained in $\mathbf{C}_{p}$. Put $G=\operatorname{Gal}(\bar{K} / K)$. Let $B_{\mathrm{DR}}$ and $B_{\text {cris }}$ be the rings introduced by Fontaine [Fo1], [Fo2]; $B_{\mathrm{DR}}$ is a complete discrete valuation field and $B_{\text {cris }}$ is a subring of $B_{\mathrm{DR}}$; by $v_{\mathrm{DR}}$, we denote the discrete valuation of $B_{\mathrm{DR}}$. We have actions of $G$ on $B_{\mathrm{DR}}$ and on $B_{\text {cris. }}$. For $i \in \mathbf{Z}$, put $B_{\mathrm{DR}}^{i}=\left\{x \in B_{\mathrm{DR}} \mid v_{\mathrm{DR}}(x) \geqq i\right\}$. Then we have $B_{\mathrm{DR}}^{i} / B_{\mathrm{DR}}^{i+1} \cong \mathbf{C}_{p}(i)$ as $G$-modules. Here $\mathbf{C}_{p}(i)$ denotes the $i$ th Tate twist.

Let $X$ be a proper smooth algebraic variety (not necessarily connected) defined over $K$. It is known that there exists a canonical isomorphism

$$
I_{\mathrm{DR}}: H_{\mathrm{et}}^{n}\left(X \times_{K} \bar{K}, \mathbf{Q}_{p}\right) \otimes_{\mathbf{Q}_{p}} B_{\mathrm{DR}} \cong H_{\mathrm{DR}}^{n}(X / K) \otimes_{K} B_{\mathrm{DR}} .
$$

The isomorphism is $B_{\mathrm{DR}}$-linear and compatible with the action of $G$ and filtrations. Taking $\mathrm{gr}_{0}$ of the filtrations, we obtain the Hodge-Tate decomposition

$$
I_{\mathrm{HT}}: H_{\mathrm{et}}^{n}\left(X \times_{K} \bar{K}, \mathbf{Q}_{p}\right) \otimes_{\mathbf{Q}_{p}} \mathbf{C}_{p} \cong \oplus_{a=0}^{n} H^{n-a}\left(X, \Omega_{X / K}^{a}\right) \otimes_{K} \mathbf{C}_{p}(-a) .
$$


Suppose that $X$ has good reduction. Let $k$ be the residue field of $K$ and $W(k)$ be the ring of Witt vectors over $k$. Let $K_{0}$ be the quotient field of $W(k)$. Then we have a canonical isomorphism ${ }^{1}$

$$
I_{\text {cris }}: H_{\text {et }}^{n}\left(X \times_{K} \bar{K}, \mathbf{Q}_{p}\right) \otimes_{\mathbf{Q}_{p}} B_{\text {cris }} \cong H_{\text {cris }}^{n}(X / W(k)) \otimes_{W(k)} B_{\text {cris }} .
$$

The isomorphism is $B_{\text {cris }}$-linear and compatible with the action of $G$ and the Frobenius map. More precisely $\sigma \in G$ acts as $\sigma \otimes \sigma$ and $1 \otimes \sigma$ on the left and the right-hand sides respectively. Concerning the Frobenius map, let $\Phi_{\text {cris }}$ be the Frobenius endomorphism of $B_{\text {cris }}$ and $\Phi$ be the Frobenius map acting on $H_{\text {cris }}^{n}(X / W(k))$. Then the action of the Frobenius map on the left-hand side is $1 \otimes \Phi_{\text {cris }}$ and $\Phi \otimes \Phi_{\text {cris }}$ on the right-hand side respectively. In good reduction case, $H_{\mathrm{DR}}^{n}(X / K)$ has a $K_{0}$-structure so that $H_{\mathrm{DR}}^{n}(X / K) \cong H_{\mathrm{DR}}^{n}\left(X / K_{0}\right) \otimes_{K_{0}}$ $K{ }^{2}$ We have another comparison isomorphism

$$
I_{0}: H_{\mathrm{cris}}^{n}(X / W(k)) \otimes_{W(k)} K_{0} \cong H_{\mathrm{DR}}^{n}\left(X / K_{0}\right)
$$

We have the relation

$$
I_{\mathrm{DR}}=\left(I_{0}\right)_{\mathrm{DR}} \circ\left(I_{\text {cris }}\right)_{\mathrm{DR}}
$$

Here $\left(I_{\text {cris }}\right)_{\mathrm{DR}}$ denotes the isomorphism obtained from (1.3) by taking $\otimes_{B_{\text {cris }}} B_{\mathrm{DR}}$ and $\left(I_{0}\right)_{\mathrm{DR}}$ denotes the isomorphism obtained from (1.4) by taking $\otimes_{K_{0}} B_{\mathrm{DR}}$.

\section{§2. The Motive Attached to an Algebraic Hecke Character}

It is possible to develop the theory of $p$-adic CM-periods using abelian varieties with complex multiplication. This approach is especially feasible in the ordinary case. However, in general, the problem of the field of definition will make it very difficult to derive precise results. Therefore it is convenient to consider motives attached to algebraic Hecke characters. In fact, even in the complex case, the precise version of Conjecture I.1.3 (cf. [Y4], p. 83, Conjecture 3.10 ) is formulated in relation to such Hecke characters. By a result of Blasius $[\mathrm{Bl} 2]$ on a $p$-adic property of Hodge classes on abelian variety, it is legitimate to transfer the results in $\S 1$ to CM-motives.

\footnotetext{
${ }^{1}$ We define $H_{\text {cris }}^{n}(X / W(k))=H_{\text {cris }}^{n}(Y / W(k))$, where $Y$ is a proper smooth algebraic variety over $k$ obtained from $X$ by reduction. By Gillet and Messing [GM], Corollary B.3.6, $H_{\text {cris }}^{n}(X / W(k))$ together with the action of the Frobenius map does not depend on the choice of $Y$.

${ }^{2}$ The $K_{0}$-structure is defined by the isomorphism of Berthelot-Ogus [BO]: $H_{\mathrm{DR}}^{n}\left(X / K_{0}\right) \cong$ $H_{\mathrm{cris}}^{n}(Y / W(k)) \otimes_{W(k)} K_{0}$, where $Y$ is the same as in footnote 1.
} 
Let $K$ be a CM-field of degree $2 n$. Let $\mathfrak{f}$ be an integral ideal of $K$. Let $\chi$ be a Grössencharacter of $K$ of conductor $\mathfrak{f}$ such that

$$
\chi((\alpha))=\prod_{\sigma \in J_{K}} \sigma(\alpha)^{l_{\sigma}}, \quad \alpha \equiv 1 \bmod ^{\times} \mathfrak{f} .
$$

Here $l_{\sigma}$ are integers such that $l_{\sigma}+l_{\sigma \rho}$ is independent of $\sigma$ and $\rho$ denotes the complex conjugation as before. Put

$$
\begin{gathered}
E=\mathbf{Q}(\chi(\mathfrak{a}) \mid \mathfrak{a} \text { is an integral ideal relatively prime to } \mathfrak{f}), \\
E_{0}=\mathbf{Q}\left(\chi((\alpha)) \mid \alpha \equiv 1 \bmod ^{\times} \mathfrak{f}\right) .
\end{gathered}
$$

Then $E$ and $E_{0}$ are algebraic number fields of finite degree; we have $E_{0} \subset E$. For $g \in \operatorname{Gal}(\overline{\mathbf{Q}} / \mathbf{Q})$, we put $l(g)=l_{g \mid K}$. Then $l$ defines a $\mathbf{Z}$-valued function on $\operatorname{Gal}(\overline{\mathbf{Q}} / \mathbf{Q})$ which is left $\operatorname{Gal}\left(\overline{\mathbf{Q}} / E_{0}\right)$-invariant and right $\operatorname{Gal}(\overline{\mathbf{Q}} / K)$-invariant.

There exists a motive $M=M(\chi)$ over $K$ with coefficients in $E ; M$ is characterized by the property ${ }^{3}$

$$
L(M, s)=(L(s, \sigma(\chi)))_{\sigma \in J_{E}}
$$

The motive $M$ has the de Rham realization $H_{\mathrm{DR}}(M)$ which is a free $E \otimes_{\mathbf{Q}} K$ module of rank $1 ; H_{\mathrm{DR}}(M)$ has the Hodge filtration $\left\{F^{m}\right\}$ which is a decreasing filtration by $E \otimes_{\mathbf{Q}} K$-submodules. Let $\psi_{m}$ be the structure function of $F^{m}$ (cf. [Y1], §2.3); $\psi_{m}$ is a $\mathbf{Z}$-valued function on $\operatorname{Gal}(\overline{\mathbf{Q}} / \mathbf{Q})$ which is left invariant under $\operatorname{Gal}(\overline{\mathbf{Q}} / E)$ and right invariant under $\operatorname{Gal}(\overline{\mathbf{Q}} / K)$. We recall the following characterization of the structure function. Let $\sigma \in J_{E}, \tau \in J_{K}$. Take $s$, $t \in \operatorname{Gal}(\overline{\mathbf{Q}} / \mathbf{Q})$ so that $\sigma=s|E, \tau=t| K$. Then the multiplicity of $\sigma$ in the left regular representation of $E$ on $F^{m} \otimes_{K, \tau} \mathbf{C}$ is equal to $\psi_{m}\left(s^{-1} t\right)$. We have (cf. [Y1], §4.2)

$$
\psi_{m}(g)= \begin{cases}1 & \text { if } l(g) \geqq m \\ 0 & \text { if } l(g)<m .\end{cases}
$$

We note that $M$ is of pure weight $l_{\sigma}+l_{\sigma \rho}, \sigma \in J_{K}$.

For a finite place $\lambda$ of $E, M$ has the $\lambda$-adic realization $H_{\lambda}(M)$ which is an $E_{\lambda}$-vector space of dimension 1 . We have an $E_{\lambda}$-linear action of $\operatorname{Gal}(\bar{K} / K)$ on $H_{\lambda}(M)$. It is related to the $p$-adic realization $H_{p}(M)$ by

$$
H_{p}(M)=\oplus_{\lambda \mid p} H_{\lambda}(M)
$$

\footnotetext{
${ }^{3}$ It is well known that $\chi$ determines the motive $M(\chi)$ up to isomorphism. See, for example, [Sc], p. 51
} 
Take $\tau_{c} \in J_{K}$. By $\tau_{c}$, we regard $M$ as a motive over $\mathbf{C}$. We have the Betti realization $H_{\tau_{c}, B}(M)$ which is an $E$-vector space of dimension 1 . We have the canonical isomorphism

$$
i_{p}: H_{\tau_{c}, B}(M) \otimes_{\mathbf{Q}} \mathbf{Q}_{p} \cong H_{p}(M)
$$

as free $E \otimes_{\mathbf{Q}} \mathbf{Q}_{p}$-modules of rank 1 .

Take $\tau_{p} \in J_{p, K}$. By $\tau_{p}$, we regard $K$ as a subfield of $\mathbf{C}_{p}$. Let $\mathfrak{P}$ be the prime ideal of $K$ induced by this embedding. We can transfer the isomorphism (1.1) to this situation and obtain the $E \otimes_{\mathbf{Q}} B_{\mathrm{DR}}$-linear isomorphism ${ }^{4}$

$$
I_{\mathrm{DR}}: H_{p}(M) \otimes_{\mathbf{Q}_{p}} B_{\mathrm{DR}} \cong H_{\mathrm{DR}}(M) \otimes_{K, \tau_{p}} B_{\mathrm{DR}} .
$$

The isomorphism is compatible with the action of $\operatorname{Gal}\left(\overline{\mathbf{Q}}_{p} / K_{\mathfrak{P}}\right)$ and filtrations.

Suppose that $\chi$ is unramified at $\mathfrak{P}$. Then $M$ has the crystalline realization $H_{\text {cris }}(M)$. (In fact, after tensoring with an Artin motive of rank 1 unramified at $\mathfrak{P}$, we may assume that $M(\chi)$ is realized as a factor of a motive generated by the motives attached to abelian varieties with complex multiplication, which are defined over $K$ and have good reduction at $\tau_{p}$. We construct the category of motives over $K$ using absolute Hodge cycles rational over $K$. Then Theorem 5.3 of Blasius, [B12] implies that an idempotent $e$ in $\operatorname{Mor}_{\mathrm{AH}}^{0}(X, X)$, in the notation of the article of Deligne-Milne in [DMOS], gives the desired crystalline realization of $(X, e)$. Here $X$ is an abelian variety defined over $K$ and have good reduction at $\tau_{p}$.) Let $k$ be the residue field of $K_{\mathfrak{P}}$ and $K_{\mathfrak{P}, 0}$ be the quotient field of $W(k)$. We identify $K_{\mathfrak{P}, 0}$ with the maximal unramified extension of $\mathbf{Q}_{p}$ contained in $K_{\mathfrak{P}}$. $H_{\text {cris }}(M)$ is a free $E \otimes_{\mathbf{Z}} W(k) \cong E \otimes_{\mathbf{Q}} K_{\mathfrak{P}, 0}$-module of rank 1. The isomorphism (1.3) transfers to the $E \otimes_{\mathbf{Q}} B_{\text {cris }}-$ linear isomorphism

$$
I_{\text {cris }}: H_{p}(M) \otimes_{\mathbf{Q}_{p}} B_{\text {cris }} \cong H_{\text {cris }}(M) \otimes_{W(k)} B_{\text {cris }} .
$$

The isomorphism is compatible with the action of $\operatorname{Gal}\left(\overline{\mathbf{Q}}_{p} / K_{\mathfrak{P}}\right)$ and the Frobenius map as noted below (1.3). Let $f$ be the degree of $\mathfrak{P}$ over $\mathbf{Q}$. We have

$$
\Phi^{f} \mid H_{\text {cris }}(M)=\chi(\mathfrak{P}) \otimes 1 .
$$

The relation (2.4) seems to be known to specialists. Since we cannot find a reference, let us give a sketch of the proof. If (2.4) holds for $M\left(\chi_{1}\right)$ and $M\left(\chi_{2}\right)$, then it holds for $M\left(\chi_{1} \chi_{2}\right)$. Therefore we may assume that the infinity type of

\footnotetext{
${ }^{4}$ Here, strictly speaking, regarding $K$ as a subfield of $\mathbf{C}_{p}$ by $\tau_{p}$, an algebraic closure of $K$ is taken inside $\mathbf{C}_{p}$. This convention will be applied hereafter, because to mention the choice of an extension of $\tau_{p}$ to an embedding of an algebraic closure of $K$ into $\mathbf{C}_{p}$ everytime is cumbersome.
} 
$\chi$ corresponds to a CM-type $\Phi$ of $K$. Let $\left(K^{\prime}, \Phi^{\prime}\right)$ be the reflex of $(K, \Phi)$. We have $E_{0}=K^{\prime}, K^{\prime} \subset E$. Let $\left(K^{\prime \prime}, \Phi^{\prime \prime}\right)$ be the reflex of $\left(K^{\prime}, \Phi^{\prime}\right)$. Then we have $K^{\prime \prime} \subset K$ and $\Phi=\operatorname{Inf}_{K / K^{\prime \prime}}\left(\Phi^{\prime \prime}\right) .{ }^{5}$ There exists an algebraic Hecke character $\chi^{\prime \prime}$ and a Hecke character of finite order $\omega$ of $K$ such that $\chi=\left(\chi^{\prime \prime} \circ N_{K / K^{\prime \prime}}\right) \times \omega$. We can choose $\chi^{\prime \prime}$ so that it is unramified at the prime divisor lying below $\mathfrak{P}$. If (2.4) holds for $M\left(\chi^{\prime \prime}\right)$, then we see easily that it also holds for $M(\chi)$. Therefore we may assume that $(K, \Phi)=\left(K^{\prime \prime}, \Phi^{\prime \prime}\right)$, replacing $\chi$ by $\chi^{\prime \prime}$ if necessary. We apply Shimura [S2], Theorem 21.4 with $\mathfrak{a}=\mathcal{O}_{K}$. Let $\tilde{\chi}$ be the corresponding Hecke character of $K_{A}^{\times}$to $\chi$. Observe that $\tilde{\chi}$ restricted to $K^{\times} U$ satisfies the conditions (19.10a) and (19.10b) of [S2], where $U$ is an open subgroup of $K_{A}^{\times}$ which contains $\mathcal{O}_{K_{\mathfrak{P}}}^{\times}$and the infinite part of $K_{A}^{\times}$. Then we see that there exist a finite abelian extension $L$ of $K$ and an abelian variety $A$ defined over $L$ with the following properties. (i) $\operatorname{End}(A) \otimes \mathbf{Q} \cong K^{\prime}, 2 \operatorname{dim} A=\left[K^{\prime}: \mathbf{Q}\right]$. (ii) $A$ is of CM-type $\left(K^{\prime}, \Phi^{\prime}\right)$ and have good reduction at every prime lying over $\mathfrak{P}$. (iii) Let $Z(s, A / L)$ be the zeta function of the motive $H^{1}(A)$, which is of rank 1 and with coefficients in $K^{\prime}$. We have $Z(s, A / L)=L\left(s, \chi \circ N_{L / K}\right)$. (iv) $\mathfrak{P}$ is unramified in $L / K$.

Put $\psi=\chi \circ N_{L / K}$. We have $H^{1}(A) \cong M(\psi)$. Since the crystalline cohomology calculates the zeta function of $A$ correctly, (2.4) holds for $H^{1}(A)$, i.e.,

$$
\Phi^{f f_{L}} \mid H_{\text {cris }}^{1}(A)=\psi(\widetilde{\mathfrak{P}}) \otimes 1,
$$

where $\widetilde{\mathfrak{P}}$ is a prime factor of $\mathfrak{P}$ in $L$ and $f_{L}$ is the relative degree of $\widetilde{\mathfrak{P}}$ over $L$. By $(2.5)$, we see that $\Phi^{f f_{L}}$ acts on the crystalline realization by $\psi(\widetilde{\mathfrak{P}})$ which can be identified with an element of $\operatorname{End}(A)$. Call it $\Sigma_{\widetilde{\mathfrak{P}}}$. Since $(K, \Phi)$ is primitive, $\Sigma_{\widetilde{\mathfrak{P}}}$ is rational over $L$ (cf. [S2], p. 65, Proposition 30). The Frobenius automorphism $\sigma_{\widetilde{\mathfrak{P}}}$ also acts on $H_{\ell}^{1}(A)$ by $\psi(\widetilde{\mathfrak{P}})=\Sigma_{\widetilde{\mathfrak{P}}} \in K^{\prime}$, for a prime $\ell \neq p$.

Now put $B=R_{L / K}(A)$, where $R_{L / K}$ denotes the restriction of scalars functor of Weil. We regard $H^{1}(B)$ as a motive over $K$ with coefficients in $E$. Then $M(\chi)$ is realized as a direct factor of $H^{1}(B)$. Let $e \in \operatorname{Mor}_{\mathrm{AH}}^{0}(B, B)$ be the idempotent such that $e H^{1}(B)=M(\chi)$. As a Galois module, $H_{\ell}^{1}(B)$ is the induced module from $H_{\ell}^{1}(A)$. From the endmorphism $\Sigma_{\widetilde{F}}$, we can obtain an endomorphism $\Sigma_{\mathfrak{P}}$ of $H^{1}(B)$ so that the action of $\sigma_{\mathfrak{P}}$ on $H_{\ell}^{1}(B)$ is given by $\Sigma_{\mathfrak{P}}$ for $\ell \neq p$. By $(2.5)$, we similarly see that the action of $\Phi^{f}$ on $H_{\text {cris }}^{1}(A)$ is given by $\Sigma_{\mathfrak{P}}$. (To see this, we first note that the case $f_{L}=1$ is easy. We may assume that $\operatorname{Gal}(L / K)$ is a cyclic extension generated by $\sigma_{\mathfrak{P}}$. By the form of

\footnotetext{
${ }^{5}$ The homomorphism $\operatorname{Inf}_{K / K^{\prime \prime}}$ from $I_{K^{\prime \prime}}$ to $I_{K}$ is defined in [S2], p. 197; $\operatorname{Inf}_{K / K^{\prime \prime}}\left(\Phi^{\prime \prime}\right)$ consists of all extensions of elements in $\Phi^{\prime \prime}$ to $J_{K}$.
} 
the induced module and using the fact that $A$ is isogenous to $\sigma(A)$ over $L$ for every $\sigma \in \operatorname{Gal}(L / K)$, we see that the action of $\sigma_{\mathfrak{P}}$ is given by the action of an (explicitly given) endomorphism $\Sigma_{\mathfrak{P}}$, which is rational over $L$. We see that $\Sigma_{\mathfrak{P}}$ is rational over $K$ using the fact that $L$ is abelian over $K$. A similar argument applies to the crystalline case with the same $\Sigma_{\mathfrak{P}}$ since $H_{\text {cris }}(B)$ is the induced module from $H_{\text {cris }}(A)$ with respect to the action of $\Phi^{f}$.) Hence we have

$$
I_{\text {cris }}\left(\Sigma_{\mathfrak{P}}(x)\right)=\Phi^{f}\left(I_{\text {cris }}(x)\right), \quad x \in H_{p}^{1}(B) .
$$

We have $e \Sigma_{\mathfrak{P}}=\chi(\mathfrak{P}) e$ on $H_{\ell}^{1}(B)$, which implies

$$
e \Sigma_{\mathfrak{P}}=\chi(\mathfrak{P}) e
$$

on $H^{1}(B)$. Now by $(2.6)$ and $(2.7)$, we have

$$
\Phi^{f} e\left(I_{\text {cris }}(x)\right)=e \Phi^{f}\left(I_{\text {cris }}(x)\right)=e I_{\text {cris }}\left(\Sigma_{\mathfrak{P}}(x)\right)=e \Sigma_{\mathfrak{P}} I_{\text {cris }}(x)=\chi(\mathfrak{P}) e I_{\text {cris }}(x)
$$

for $x \in H_{p}^{1}(B)$. Hence (2.4) follows.

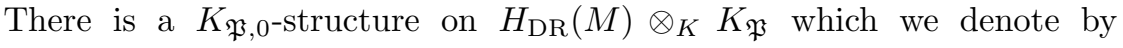
$H_{\mathrm{DR}}\left(M / K_{\mathfrak{P}, 0}\right)$. The isomorphism (1.4) transfers to

$$
I_{0}: H_{\text {cris }}(M) \cong H_{\mathrm{DR}}\left(M / K_{\mathfrak{P}, 0}\right) \text {. }
$$

\section{§3. Definition of the $p$-Adic Period}

Let $K, \chi$ and $M=M(\chi)$ be the same as in $\S 2$. We choose $\tau_{c} \in J_{K}$ and $\tau_{p} \in J_{p, K}$. Let $\mathfrak{P}$ be the prime ideal of $K$ determined by $\tau_{p}$.

Now take $0 \neq c_{B} \in H_{\tau_{c}, B}(M)$. Then $i_{p}\left(c_{B}\right)$ is a generator of $H_{p}(M)$ as an $E \otimes_{\mathbf{Q}} \mathbf{Q}_{p}$-module. Take $c_{\mathrm{DR}} \in H_{\mathrm{DR}}(M)$ which is a generator of $H_{\mathrm{DR}}(M)$ as an $E \otimes_{\mathbf{Q}} K$-module. Then we define the period $P\left(\chi ; \tau_{c}, \tau_{p}\right)$ by

$$
I_{\mathrm{DR}}\left(i_{p}\left(c_{B}\right) \otimes 1\right)=P\left(\chi ; \tau_{c}, \tau_{p}\right)\left(c_{\mathrm{D} R} \otimes 1\right) .
$$

We have $P\left(\chi ; \tau_{c}, \tau_{p}\right) \in\left(E \otimes_{\mathbf{Q}} B_{\mathrm{DR}}\right)^{\times}$; it is determined up to multiplication of elements of $\left(E \otimes_{\mathbf{Q}} K\right)^{\times}$. Here we regard $E \otimes_{\mathbf{Q}} K$ as a subring of $E \otimes_{\mathbf{Q}} B_{\mathrm{DR}}$ by $a \otimes b \rightarrow a \otimes \tau_{p}(b)$. When no confusion is likely, we abbreviate $P\left(\chi ; \tau_{c}, \tau_{p}\right)$ to $P(\chi)$.

We assume that $\chi$ is unramified at $\mathfrak{P}$. We assume that

$$
c_{\mathrm{DR}} \text { belongs to } H_{\mathrm{DR}}\left(M / K_{\mathfrak{P}, 0}\right) \text {. }
$$

This condition is satisfied if $K_{\mathfrak{P}}=K_{\mathfrak{P}, 0}$. Then we can choose a generator $c_{\text {cris }}$ of $H_{\text {cris }}(M)$ as an $E \otimes_{\mathbf{Z}} W(k)$-module so that

$$
I_{0}\left(c_{\text {cris }} \otimes 1\right)=c_{\mathrm{DR}} \otimes 1 .
$$


We define $\widetilde{P}\left(\chi ; \tau_{c}, \tau_{p}\right) \in\left(E \otimes_{\mathbf{Q}} B_{\text {cris }}\right)^{\times}$by

$$
I_{\text {cris }}\left(i_{p}\left(c_{B}\right) \otimes 1\right)=\widetilde{P}\left(\chi ; \tau_{c}, \tau_{p}\right)\left(c_{\text {cris }} \otimes 1\right) .
$$

Since $B_{\text {cris }} \subset B_{\mathrm{DR}}$, we can regard $\widetilde{P}\left(\chi ; \tau_{c}, \tau_{p}\right) \in\left(E \otimes_{\mathbf{Q}} B_{\mathrm{DR}}\right)^{\times}$. Then, by $(1.5)$ and (3.2), we have

$$
P\left(\chi ; \tau_{c}, \tau_{p}\right)=\widetilde{P}\left(\chi ; \tau_{c}, \tau_{p}\right)
$$

In view of this relation, we write $\widetilde{P}\left(\chi ; \tau_{c}, \tau_{p}\right)$ as $P\left(\chi ; \tau_{c}, \tau_{p}\right)$, or for simplicity as $P(\chi)$. (The $p$-adic period in the standard sense is $P(\chi)$. We can make $B_{\mathrm{DR}}$ descend to $B_{\text {cris }}$, which is a crucial observation since the Frobenius endomorphism $\Phi_{\text {cris }}$ can act on $B_{\text {cris }}$.)

Now we apply the Frobenius map on the both sides of (3.3). We have

$$
I_{\text {cris }}\left(i_{p}\left(c_{B}\right) \otimes 1\right)=\Phi_{\text {cris }}(P(\chi))\left(\Phi\left(c_{\text {cris }}\right) \otimes 1\right) .
$$

Repeating this operation $f$ times and using (2.4), we get

$$
I_{\text {cris }}\left(i_{p}\left(c_{B}\right) \otimes 1\right)=\Phi_{\text {cris }}^{f}(P(\chi))\left(\chi(\mathfrak{P}) c_{\text {cris }} \otimes 1\right) .
$$

Comparing with (3.3), we obtain

$$
\Phi_{\text {cris }}^{f}(P(\chi))(\chi(\mathfrak{P}) \otimes 1)=P(\chi) .
$$

We state two formal consequences of the existence of the motive $M(\chi)$. Let $\chi_{1}$ and $\chi_{2}$ be Grössencharacters of $K$ of the type (2.1). Let $E$ be an algebraic number field which contains the values of $\chi_{1}$ and $\chi_{2}$. We regard $M\left(\chi_{i}\right)$ as a motive over $K$ with coefficients in $E$. Then since $M\left(\chi_{1}\right) \otimes_{E} M\left(\chi_{2}\right)=M\left(\chi_{1} \chi_{2}\right)$, we obtain

$$
P\left(\chi_{1} \chi_{2}\right) \equiv P\left(\chi_{1}\right) P\left(\chi_{2}\right) \quad \bmod \left(E \otimes_{\mathbf{Q}} K\right)^{\times}
$$

Next let $L$ be a CM-field which contains $K$. Since the extension of scalars of $M(\chi)$ to $L$ is $M\left(\chi \circ N_{L / K}\right)$, we have

$$
P\left(\chi ; \tau_{c}, \tau_{p}\right) \equiv P\left(\chi \circ N_{L / K} ; \tilde{\tau}_{c}, \tilde{\tau}_{p}\right) \bmod \left(E \otimes_{\mathbf{Q}} L\right)^{\times} .
$$

Here $\tilde{\tau}_{c} \in J_{L}, \tilde{\tau}_{p} \in J_{p, L}$ denote (any) extensions of $\tau_{c}$ and $\tau_{p}$ respectively. 


\section{$\S 4$. The Invariant $Q$}

In this section, we will examine the $p$-adic period $P(\chi)$ in more detail. We fix an embedding $\overline{\mathbf{Q}} \subset \mathbf{C}_{p}$. Recall our convention that $\overline{\mathbf{Q}}$ is a subfield of $\mathbf{C}$. Take $\tau_{c}=\mathrm{id}, \tau_{p}=\mathrm{id}$ in (3.1). Since $\overline{\mathbf{Q}}_{p} \subset B_{\mathrm{DR}}$, we have an isomorphism

$$
E \otimes_{\mathbf{Q}} B_{\mathrm{DR}} \cong \oplus_{\sigma \in J_{p, E}} B_{\mathrm{DR}}
$$

We write

$$
P(\chi)=(P(\chi)(\sigma))_{\sigma \in J_{p, E}}
$$

according to this decomposition. Take $\sigma \in J_{p, E}$ and $s \in \operatorname{Gal}(\overline{\mathbf{Q}} / \mathbf{Q})$ such that $s \mid E=\sigma$. By (2.2) and [Y1], Lemma 2.1, (1), we see that the $\sigma$-component of $F^{m} \otimes_{K, \text { id }} \overline{\mathbf{Q}_{p}}$ is nonzero for $m \leqq l\left(s^{-1}\right)$ and zero for $m>l\left(s^{-1}\right)$. Since $I_{\mathrm{DR}}$ preserves the filtrations, we have

$$
v_{\mathrm{DR}}(P(\chi)(\sigma))=-l\left(s^{-1}\right) .
$$

Since $H_{\text {cris }}(M, W(k)) \otimes_{W(k)} K_{\mathfrak{P}, 0}$ is a free $E \otimes_{\mathbf{Q}} K_{\mathfrak{P}, 0}$-module of rank 1, we can write

$$
\Phi^{i}\left(c_{\text {cris }} \otimes 1\right)=Q^{(i)}\left(c_{\text {cris }} \otimes 1\right), \quad 1 \leqq i \in \mathbf{Z}
$$

with $Q^{(i)} \in\left(E \otimes_{\mathbf{Q}} K_{\mathfrak{P}, 0}\right)^{\times}$. Applying the Frobenius map $i$-times on (3.3), we obtain

$$
\Phi_{\text {cris }}^{i}(P(\chi)) \Phi^{i}\left(c_{\text {cris }} \otimes 1\right)=P(\chi)\left(c_{\text {cris }} \otimes 1\right) .
$$

Therefore we obtain

$$
\Phi_{\text {cris }}^{i}(P(\chi)) Q^{(i)}=P(\chi), \quad 1 \leqq i \in \mathbf{Z} .
$$

We put $Q=Q^{(1)}$. Then we have

$$
\Phi_{\text {cris }}(P(\chi)) Q=P(\chi) .
$$

Let $\mathbf{Q}_{p}^{\mathrm{ur}}$ be the maximal unramified extension of $\mathbf{Q}_{p}$ and let $\varphi \in \operatorname{Gal}\left(\mathbf{Q}_{p}^{\mathrm{ur}} / \mathbf{Q}_{p}\right)$ be the absolute Frobenius map. Applying $\Phi_{\text {cris }}$ on both sides of (4.4) noting that $\Phi_{\text {cris }}$ acts on $K_{\mathfrak{P}, 0}$ by the absolute Frobenius map $\varphi$, we get

$$
\Phi_{\text {cris }}^{2}(P(\chi)) \varphi(Q)=\Phi_{\text {cris }}(P(\chi))=P(\chi) Q^{-1} .
$$

Hence we have

$$
Q^{(2)}=\varphi(Q) Q
$$


Repeating this process, we obtain the relation

$$
Q^{(i)}=\varphi^{i-1}(Q) \cdots \varphi(Q) Q, \quad 1 \leqq i \in \mathbf{Z}
$$

which can also be derived directly from (4.2). We note that

$$
Q^{(f)}=\chi(\mathfrak{P}) \otimes 1 \in\left(E \otimes_{\mathbf{Q}} K_{\mathfrak{P}, 0}\right)^{\times}
$$

which follows from (3.5) (or directly from (2.4)).

\section{§5. The Main Conjecture}

Let $K$, an embedding $K \subset \mathbf{C}_{p}, \mathfrak{P}$ and $\chi$ be the same as before. We assume that $\chi$ is unramified at $\mathfrak{P}$. Suppose that the CM-field $K$ is abelian over a totally real number field $F$ as in our construction of $l g_{p, K / F}$. Using $Q^{(i)}$, we can predict the nature of $l g_{p, K / F}$ in the general case. Take $\tau \in \operatorname{Gal}(K / F)$. We have

$$
Q^{(i)} \in\left(E \otimes_{\mathbf{Q}} K_{\mathfrak{P}, 0}\right)^{\times} \subset\left(E \otimes_{\mathbf{Q}} \overline{\mathbf{Q}_{p}}\right)^{\times} \cong \prod_{\sigma \in J_{p, E}}{\overline{\mathbf{Q}_{p}}}^{\times}
$$

Let $Q^{(i)}(\sigma) \in{\overline{\mathbf{Q}_{p}}}^{\times}$denote the $\sigma$-component of $Q^{(i)}$ with respect to this decomposition. Let $\mathfrak{p}$ be the prime ideal of $F$ lying below $\mathfrak{P}$ and let $f_{0}$ be the degree of $\mathfrak{p}$ over $\mathbf{Q}$. We take a Hecke character $\chi$ of the form

$$
\chi((\alpha))=(\rho \tau(\alpha) / \tau(\alpha))^{l}, \quad \alpha \equiv 1 \quad \bmod ^{\times} \mathfrak{f}
$$

with $1 \leqq l \in \mathbf{Z}$. We can take $l$ and $\chi$ so that $E \subset K$.

Conjecture $\mathbf{Q}$. We assume $(\mathrm{A})$ and that the prime ideal $\mathfrak{p}$ is unramified in $K$. Then we have

$$
l g_{p, K / F}\left(\mathrm{id}, \tau^{-1}\right)=\frac{1}{2 l} \log _{p} Q^{\left(f_{0}\right)}(\mathrm{id})+a \log _{p} b
$$

with $a \in \mathbf{Q}, b \in K^{\times}$.

Since $P(\chi)$ is determined up to multiplication by elements in $\left(E \otimes_{\mathbf{Q}}\left(K \cap K_{\mathfrak{P}, 0}\right)\right)^{\times}, Q^{\left(f_{0}\right)}$ is determined up to multiplication by elements of the form $\varphi^{f_{0}}(c) / c, c \in\left(E \otimes_{\mathbf{Q}}\left(K \cap K_{\mathfrak{P}, 0}\right)\right)^{\times}$by (4.3). Here $\varphi$ acts on $E \otimes_{\mathbf{Q}}\left(K \cap K_{\mathfrak{P}, 0}\right)$ through the second factor. Therefore the validity of Conjecture $\mathrm{Q}$ does not depend on the choices of $c_{B}, c_{\mathrm{cris}}$ and $c_{\mathrm{DR}}$. 
Suppose that $\mathfrak{p}$ splits completely in $K$. Then $f_{0}=f$ and we have $Q^{(f)}=$ $\chi(\mathfrak{P}) \otimes 1$ by (4.6). Since $\varphi^{f}$ acts trivially on $K_{\mathfrak{P}, 0}, Q^{(f)}$ is determined independently of the choices of $c_{B}, c_{\text {cris }}$ and $c_{\mathrm{DR}}$. Put $\mathfrak{P}^{h_{K}}=(\alpha)$. Take a positive integer $m$ so that $\alpha^{m} \equiv 1 \bmod \mathfrak{f}$. Then we have

$$
\frac{1}{2 l} \log _{p} Q^{\left(f_{0}\right)}(\mathrm{id})=\frac{1}{2 l m h_{K}} \log _{p}\left(\rho \tau\left(\alpha^{m}\right)^{l} / \tau\left(\alpha^{m}\right)^{l}\right)=\frac{1}{2 h_{K}} \log _{p}(\rho \tau(\alpha) / \tau(\alpha)) .
$$

Thus, in this case, Conjecture I.A implies that Conjecture Q holds with an explicitly given term $a \log _{p} b$.

Remark 1. Ogus [O] defined $Q^{(i)}$ using results in Berthelot-Ogus [BO] on crystalline cohomology. According to his results in $[\mathrm{O}]$, we can show that Conjecture $\mathrm{Q}$ is true when $F=\mathbf{Q}$ and $K=\mathbf{Q}\left(\zeta_{n}\right)$ with $\zeta_{n}$ a primitive $n$th root of unity, $(p, n)=1$. We can apply similar arguments as in [Y4], Chapter III, $\S 2$ as follows. In this Remark, we put $L Q(\tau)=\frac{1}{2 l} \log _{p} Q^{(1)}($ id) for $\tau \in \operatorname{Gal}(K / F)$ and for an algebraic Hecke character as in Conjecture Q. We define $\sigma(a) \in$ $\operatorname{Gal}\left(\mathbf{Q}\left(\zeta_{n}\right) / \mathbf{Q}\right)$ for $a \in(\mathbf{Z} / n \mathbf{Z})^{\times}$by $\zeta_{n}^{\sigma(a)}=\zeta_{n}^{a}$. Let $r, s, t$ be integers satisfying $0<r, s, t<n, r+s+t=n,(r, s, t, n)=1$. Then $\Phi_{r, s, t}=\left\{\sigma(a) \mid a \in H_{r, s, t}\right\}$ with $H_{r, s, t}=\left\{a \in(\mathbf{Z} / n \mathbf{Z})^{\times}|\langle\mid a r\rangle\rangle+\langle\langle a s\rangle\rangle+\langle\langle a t\rangle\rangle=n\right\}$ is a CM-type of $\mathbf{Q}\left(\zeta_{n}\right)$. Here for $a \in \mathbf{Z} / n \mathbf{Z}$, we denote by $\langle\langle a\rangle\rangle$ an integer which satisfies $0 \leq\langle\langle a\rangle\rangle\langle n$, $\langle\langle a\rangle\rangle \equiv a \bmod n$. We consider the Fermat curve $F_{n}: x^{n}+y^{n}=1$ and a differential form $\eta_{r, s, t}=x^{r-1} y^{s-n} d x$ on $F_{n}$. Let $A_{r, s, t}$ be the factor of the Jacobian variety of $F_{n}$ which corresponds to differential forms $\left\{\eta_{\langle a r\rangle\rangle,\langle\langle a s\rangle\rangle,\langle\langle a t\rangle\rangle} \mid\right.$ $\left.a \in H_{r, s, t}\right\}$. Then $A_{r, s, t}$ is an abelian variety defined over $\mathbf{Q}$, which is of CM-type $\left(\mathbf{Q}\left(\zeta_{n}\right), \Phi_{r, s, t}\right)$. We denote the algebraic Hecke character of $\mathbf{Q}\left(\zeta_{n}\right)$ associated to $A_{r, s, t}$ by $\chi_{r, s, t}$. Then $\chi_{r, s, t}$ satisfies that $l_{\sigma}=1$ if $\sigma^{-1} \in \Phi_{r, s, t},=0$

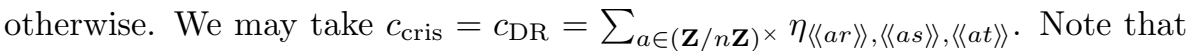
$\eta_{r, s, t}$ is equal to $\xi_{-\frac{r}{n},-\frac{s}{n},-\frac{t}{n}}$ in [O]. By Proposition 2.4 in [O], for $a \in(\mathbf{Z} / n \mathbf{Z})^{\times}$ we have

$$
\log _{p} Q^{(1)}\left(\chi_{r, s, t}\right)(\sigma(a)) \equiv \log _{p}\left(\frac{\Gamma_{p}\left(\frac{\langle a r\rangle\rangle}{n}\right) \Gamma_{p}\left(\frac{\langle a s s\rangle}{n}\right)}{\Gamma_{p}\left(\frac{\langle a r\rangle\rangle+\langle\langle a s\rangle\rangle}{n}\right)}\right) \quad \bmod \mathbf{Q} \log _{p} \mathbf{Q}\left(\zeta_{n}\right)^{\times} .
$$

It gives a $p$-adic counterpart of (2.3) in [Y4], Chapter III. It also gives

$$
\log _{p} Q^{(1)}\left(\chi_{r, s, t}\right)(\sigma(a))+\log _{p} Q^{(1)}\left(\chi_{r, s, t}\right)(\sigma(-a)) \equiv 0 \quad \bmod \mathbf{Q} \log _{p} \mathbf{Q}\left(\zeta_{n}\right)^{\times} .
$$

Note that we have $Q(\chi)^{(i)}(\tau \sigma) \equiv Q(\tau \circ \chi)^{(i)}(\sigma)$ for any $\chi, i, \tau, \sigma$ since we may identify the $\tau \sigma$-component of $H_{\text {cris }}(M(\chi)) \otimes_{K_{\mathfrak{P}, 0}} \overline{\mathbf{Q}_{p}}$ and the $\sigma$-component of $H_{\text {cris }}(M(\tau \circ \chi)) \otimes_{K_{\mathfrak{P}, 0}} \overline{\mathbf{Q}_{p}}$ (cf. (8.2)). Therefore we can write

$$
\log _{p} Q^{(1)}\left(\chi_{r, s, t}\right)(\mathrm{id}) \equiv \sum_{\sigma \in \Phi_{r, s, t}} L Q\left(\sigma^{-1}\right) \quad \bmod \mathbf{Q} \log _{p} \mathbf{Q}\left(\zeta_{n}\right)^{\times} .
$$


By the same arguments as for (2.5) in [Y4], Chapter III, we have

$$
\sum_{t=1,(t, n)=1}^{[(n-1) / 2]} \epsilon_{a t} L Q\left(\sigma(t)^{-1}\right) \equiv \log _{p}\left(\frac{\Gamma_{p}\left(\frac{a}{n}\right) \Gamma_{p}\left(\frac{1}{n}\right)}{\Gamma_{p}\left(\frac{a+1}{n}\right)}\right) \quad \bmod \mathbf{Q} \log _{p} \mathbf{Q}\left(\zeta_{n}\right)^{\times}
$$

where $a \in(\mathbf{Z} / n \mathbf{Z})^{\times}, T_{a}=\left\{t \in(\mathbf{Z} / n \mathbf{Z})^{\times} \mid \frac{\langle\langle a t\rangle\rangle}{n}+\frac{\langle\langle t\rangle\rangle}{n}<1\right\}, \epsilon_{a t}=1$ if $t \in T_{a}$, $=-1$ otherwise. Other arguments are the same as for the proof of Theorem 2.6 in [Y4], Chapter III if we replace $\Gamma, L(s, \eta)$ by $\Gamma_{p}, L_{p}(s, \omega \eta)$ respectively. Let $K$ a CM-subfield of $\mathbf{Q}\left(\zeta_{n}\right)$. For $\sigma \in G=\operatorname{Gal}(K / \mathbf{Q})$, we have

$$
L Q\left(\sigma^{-1}\right) \equiv \sum_{\eta \in \hat{G}_{-}} \frac{\eta(\sigma)}{[K: \mathbf{Q}]} \frac{L_{p}^{\prime}(0, \omega \eta)}{L(0, \eta)} \bmod \mathbf{Q} \log _{p} \mathbf{Q}\left(\zeta_{n}\right)^{\times}
$$

Although we do not give the details here, we can replace $\bmod \mathbf{Q} \log _{p} \mathbf{Q}\left(\zeta_{n}\right)^{\times}$ by $\bmod \mathbf{Q} \log _{p} K^{\times}$by further computations.

Remark 2. In the $p$-adic case, the nature of $l g_{p, K / F}(\mathrm{id}, \tau)$ would depend heavily on the choice of $F$. This is the main difference from the complex case.

Remark 3. Using (3.6), it is easy to see that the left-hand side of Conjecture $\mathrm{Q}$, taken modulo $\mathrm{Q} \log _{p} K^{\times}$, is independent of the choice of $l$ and $\chi$.

\section{§6. Toward the Understanding of the $p$-Adic Period}

Conjecture $\mathrm{Q}$ in the previous section gives information on the $Q$-invariant. We will investigate the $p$-adic period itself in relation to this conjecture. We will see that, in the ordinary case, the $p$-adic period can be recovered from the $Q$-invariant modulo $\mathbf{Q}_{p}^{\times}$in the next section.

Let $v$ be the additive valuation of $\mathbf{C}_{p}$ normalized by $v(p)=1$. We fix an embedding $\overline{\mathbf{Q}} \subset \mathbf{C}_{p}$. Let $K$ be a CM-field and $\mathfrak{P}$ be the prime ideal of $K$ obtained from the embedding $K \subset \overline{\mathbf{Q}} \subset \mathbf{C}_{p}$ as before. Let $e$ and $f$ be the ramification index and the degree of $\mathfrak{P}$ over $\mathbf{Q}$ respectively. We recall our notations. We take a Hecke character $\chi$ of $K$ satisfying (2.1). We assume that $\chi$ is unramified at $\mathfrak{P}$. The $p$-adic period $P(\chi)=\widetilde{P}(\chi ;$ id, id $) \in\left(E \otimes_{\mathbf{Q}} B_{\text {cris }}\right)^{\times}$ is defined by (3.3). The $Q$-invariant $Q=Q^{(1)} \in\left(E \otimes_{\mathbf{Q}} K_{\mathfrak{P}, 0}\right)^{\times}$is defined by (4.2).

Lemma 6.1. Put $G=\operatorname{Gal}(\overline{\mathbf{Q}} / \mathbf{Q}), H=\operatorname{Gal}(\overline{\mathbf{Q}} / K)$. Let $\widetilde{\mathfrak{P}}$ be the prime divisor of $\overline{\mathbf{Q}}$ induced by the embedding $\overline{\mathbf{Q}} \subset \mathbf{C}_{p}$ and let $Z$ be the decomposition 
group of $\widetilde{\mathfrak{P}}$. For $\sigma \in J_{p, E}$, we take $s \in \operatorname{Gal}(\overline{\mathbf{Q}} / \mathbf{Q})$ so that $s \mid E=\sigma$. Then we have

$$
v(\sigma(\chi(\mathfrak{P})))=\frac{1}{e} \sum_{\tau \in Z H / H} l\left(s^{-1} \tau\right)
$$

Proof. Take $0<m \in \mathbf{Z}$ so that $\mathfrak{P}^{m}=(\alpha), \alpha \in \mathcal{O}_{K}, \alpha \equiv 1 \bmod \mathfrak{f}$. Since

$$
\chi(\mathfrak{P})^{m}=\prod_{\tau \in J_{K}} \tau(\alpha)^{l_{\tau}} \in E,
$$

we have

$$
v(\sigma(\chi(\mathfrak{P})))=\frac{1}{m} v\left(s \prod_{\tau \in G / H} \tau(\alpha)^{l(\tau)}\right)=\frac{1}{m} v\left(\prod_{\tau \in G / H} \tau(\alpha)^{l\left(s^{-1} \tau\right)}\right) .
$$

Since

$$
v(\tau(\alpha))>0 \Longleftrightarrow \tau \in Z H,
$$

we have

$$
v(\sigma(\chi(\mathfrak{P})))=\frac{1}{m} v\left(\prod_{\tau \in Z H / H} \tau(\alpha)^{l\left(s^{-1} \tau\right)}\right) .
$$

For $\tau \in Z H / H$, we have $v(\tau(\alpha))=m / e$. Hence the assertion follows.

Corollary 1. If $\mathfrak{P}$ is of degree 1 and unramified over $\mathbf{Q}$, i.e., $e=f=1$, then we have

$$
v(\sigma(\chi(\mathfrak{P})))=l\left(s^{-1}\right) .
$$

The assertion is obvious since $Z \subset H$.

Corollary 2. If $p$ splits completely in $E_{0}$, then we have $v(\sigma(\chi(\mathfrak{P})))=$ $f l\left(s^{-1}\right)$.

Proof. Put $H^{\prime}=\operatorname{Gal}\left(\overline{\mathbf{Q}} / E_{0}\right)$. Since $p$ splits completely in $E_{0}$, we have

$$
g^{-1} Z g \subset H^{\prime} \quad \text { for all } g \in G \text {. }
$$

Since the function $l$ is left $H^{\prime}$-invariant and right $H$-invariant, we obtain, from (6.1),

$$
v(\sigma(\chi(\mathfrak{P})))=\frac{l\left(s^{-1}\right)}{e}[Z H: H]=\frac{l\left(s^{-1}\right)}{e}[Z: H \cap Z]=\frac{l\left(s^{-1}\right)}{e}\left[K_{\mathfrak{P}}: \mathbf{Q}_{p}\right] .
$$

This completes the proof. 
Hereafter until the end of $\S 7$, we assume that $p$ is unramified in $E$. Let $\mathbf{Q}_{p}^{\text {ur }}$ be the maximal unramified extension of $\mathbf{Q}_{p}$ and $\widehat{\mathbf{Q}_{p}^{\text {ur }}}$ be the closure of $\mathbf{Q}_{p}^{\text {ur }}$ in $\mathbf{C}_{p}$. Let $\varphi \in \operatorname{Gal}\left(\overline{\mathbf{Q}_{p}} / \mathbf{Q}_{p}\right)$ be a Frobenius map which gives the absolute Frobenius automorphism of $\operatorname{Gal}\left(\mathbf{Q}_{p}^{\text {un }} / \mathbf{Q}_{p}\right)$. We use the same letter $\varphi$ for the extension of $\varphi \mid \operatorname{Gal}\left(\mathbf{Q}_{p}^{\text {un }} / \mathbf{Q}_{p}\right)$ to the continuous automorphism of $\widehat{\mathbf{Q}_{p}^{\text {ur }}}$. Since $\sigma(E) \subset \mathbf{Q}_{p}^{\text {ur }}$ for every $\sigma \in J_{p, E}$, we have

$$
E \otimes_{\mathbf{Q}} \widehat{\mathbf{Q}_{p}^{\mathrm{ur}}} \cong \oplus_{\sigma \in J_{p, E}} \widehat{\mathbf{Q}_{p}^{\mathrm{ur}}}
$$

We write $x \in E \otimes_{\mathbf{Q}} \widehat{\mathbf{Q}_{p}^{\text {ur }}}$ as $x=(x(\sigma))_{\sigma \in J_{p, E}}$ according to this decomposition. Similarly, since $\widehat{\mathbf{Q}_{p}^{\text {ur }}} \subset B_{\text {cris }}$, we have

$$
E \otimes_{\mathbf{Q}} B_{\text {cris }} \cong \oplus_{\sigma \in J_{p, E}} B_{\text {cris }}
$$

We write $x \in E \otimes_{\mathbf{Q}} B_{\text {cris }}$ as $x=(x(\sigma))_{\sigma \in J_{p, E}}$ according to this decomposition.

Lemma 6.2. Let $x=(x(\sigma))_{\sigma \in J_{p, E}} \in E \otimes_{\mathbf{Q}} \widehat{\mathbf{Q}_{p}^{u r}}, y=(y(\sigma))_{\sigma \in J_{p, E}}=$ $(1 \otimes \varphi)(x) \in E \otimes_{\mathbf{Q}} \widehat{\mathbf{Q}_{p}^{\text {ur }}}$. For $\sigma \in J_{p, E}$, define $\sigma_{1} \in J_{p, E}$ by $\sigma_{1}\left|E=\varphi^{-1} \sigma\right| E$. Then we have $y(\sigma)=\varphi\left(x\left(\sigma_{1}\right)\right)$. Similarly for $x=(x(\sigma))_{\sigma \in J_{p, E}} \in E \otimes_{\mathbf{Q}} B_{\text {cris }}$ and $y=(y(\sigma))_{\sigma \in J_{p, E}}=\left(1 \otimes \Phi_{\text {cris }}\right)(x) \in E \otimes_{\mathbf{Q}} B_{\text {cris }}$, we have $y(\sigma)=\Phi_{\text {cris }}\left(x\left(\sigma_{1}\right)\right)$.

Proof. Here we prove only the first assertion. The proof of the second assertion is similar. We write $E=\mathbf{Q}(\theta)$ and let $f(X)$ be the minimal polynomial of $\theta$ over $\mathbf{Q}$. In $\mathbf{Q}_{p}^{\mathrm{ur}}[X]$, we can decompose $f(X)$ as

$$
f(X)=\prod_{\sigma \in J_{p, E}}(X-\sigma(\theta)) .
$$

For $h \in \mathbf{Q}[X]$ and $a \in \widehat{\mathbf{Q}_{p}^{\text {ur }}}, h(\theta) \otimes a$ is mapped to $(h(\sigma(\theta)) a)_{\sigma \in J_{p, E}}$ and $(1 \otimes \varphi)(h(\theta) \otimes a)$ is mapped to $(h(\sigma(\theta)) \varphi(a))_{\sigma \in J_{p, E}}$ by the isomorphism (6.2). It suffices to prove the relation when $x(\sigma)=h(\sigma(\theta)) a, y(\sigma)=h(\sigma(\theta)) \varphi(a)$, $\sigma \in J_{p, E}$. Now we have

$$
\varphi\left(x\left(\sigma_{1}\right)\right)=h\left(\varphi \sigma_{1}(\theta)\right) \varphi(a)=h(\sigma(\theta)) \varphi(a)=y(\sigma)
$$

and the proof is complete.

Lemma 6.3. If $x \in \widehat{\mathbf{Q}_{p}^{\text {ur }}}$ satisfies $v(x)=0$, then there exists $y \in \widehat{\mathbf{Q}_{p}^{\text {ur }}}$ such that $\varphi(y) y^{-1}=x$. Moreover $y$ is unique up to multiplication by elements in $\mathbf{Q}_{p}^{\times}$. 
Proof. Let $\overline{\mathbf{F}_{p}}$ be an algebraic closure of $\mathbf{F}_{p}=\mathbf{Z} / p \mathbf{Z}$ and let $A$ be the ring of integers of $\widehat{\mathbf{Q}_{p}^{\text {ur }}}$. Then $(p)$ is the maximal ideal of $A$. We fix an isomorphism $A /(p) \cong \overline{\mathbf{F}_{p}}$. Let $\iota$ be an injection of $\overline{\mathbf{F}_{p}}$ into $A$ such that $\iota(a) \bmod p=a$, $\varphi(\iota(a))=\iota\left(a^{p}\right)$. An element of $\widehat{\mathbf{Q}_{p}^{\text {ur }}}$ can be written as $\sum_{n=n_{0}}^{\infty} \iota\left(a_{n}\right) p^{n}, a_{n} \in \overline{\mathbf{F}_{p}}$, $n_{0} \in \mathbf{Z}$. Now put $x=\sum_{n=0}^{\infty} \iota\left(\alpha_{n}\right) p^{n}, \alpha_{n} \in \overline{\mathbf{F}_{p}}, \alpha_{0} \neq 0, y=\sum_{n=0}^{\infty} \iota\left(\beta_{n}\right) p^{n}$, $\beta_{n} \in \overline{\mathbf{F}_{p}}$. Then $\varphi(y) y^{-1}=x$ is equivalent to

$$
\left(\sum_{n=0}^{\infty} \iota\left(\alpha_{n}\right) p^{n}\right)\left(\sum_{n=0}^{\infty} \iota\left(\beta_{n}\right) p^{n}\right)=\sum_{n=0}^{\infty} \iota\left(\beta_{n}^{p}\right) p^{n} .
$$

If we consider the equation $(*)$ modulo $p$, we have $\alpha_{0} \beta_{0}=\beta_{0}^{p}$. We choose $\beta_{0} \in{\overline{\mathbf{F}_{p}}}^{\times}$so that $\beta_{0}^{p-1}=\alpha_{0}$. Now let $N \geqq 1$ and assume that $(*)$ holds modulo $p^{N}$ with $\beta_{0}, \beta_{1}, \ldots, \beta_{N-1}$. Put

$$
c=\left(\sum_{n=0}^{N-1} \iota\left(\alpha_{n}\right) p^{n}\right)\left(\sum_{n=0}^{N-1} \iota\left(\beta_{n}\right) p^{n}\right)-\sum_{n=0}^{N-1} \iota\left(\beta_{n}^{p}\right) p^{n}
$$

and take $\gamma \in \overline{\mathbf{F}_{p}}$ so that $\gamma=\left(c / p^{N}\right) \bmod p$. The condition that $(*)$ holds modulo $p^{N+1}$ is

$$
\beta_{N}^{p}=\sum_{n=0}^{N} \alpha_{n} \beta_{N-n}+\gamma .
$$

This is an equation of Artin-Schreier type with respect to $\beta_{N}$ and we can find a solution $\beta_{N} \in \overline{\mathbf{F}_{p}}$. Hence the existence of $y$ follows. The uniqueness assertion is clear. This completes the proof.

We write $Q \in\left(E \otimes_{\mathbf{Q}} K_{\mathfrak{P}, 0}\right)^{\times} \subset\left(E \otimes_{\mathbf{Q}} \widehat{\mathbf{Q}_{p}^{\text {ur }}}\right)^{\times}$as $Q=(Q(\sigma))_{\sigma \in J_{p, E}}$. Taking the $\sigma$-component of (4.4), we obtain

$$
\left(\Phi_{\text {cris }}(P(\chi))\right)(\sigma) Q(\sigma)=P(\chi)(\sigma) .
$$

Let $t \in B_{\text {cris }}$ be an element such that $\Phi_{\text {cris }}(t)=p t$ and that $t$ is a uniformizer of $B_{\mathrm{DR}}$. For $\sigma \in J_{p, E}$, we take $s \in \operatorname{Gal}(\overline{\mathbf{Q}} / \mathbf{Q})$ so that $s \mid E=\sigma$. Put

$$
P(\chi)(\sigma)=X(\sigma) \cdot t^{-l\left(s^{-1}\right)}
$$

with $X(\sigma) \in B_{\text {cris }}$. By (4.1), we have $v_{\mathrm{DR}}(X(\sigma))=0$. By Lemma 6.2, we have

$$
\left(\Phi_{\text {cris }}(P(\chi))\right)(\sigma)=\Phi_{\text {cris }}\left(X\left(\sigma_{1}\right)\right) p^{-l\left(s_{1}^{-1}\right)} \cdot t^{-l\left(s_{1}^{-1}\right)},
$$

where $s_{1}=\varphi^{-1} s$ and $\sigma_{1}=s_{1} \mid E$. By (6.4), we obtain

$$
\Phi_{\text {cris }}\left(X\left(\sigma_{1}\right)\right) p^{-l\left(s_{1}^{-1}\right)} \cdot t^{-l\left(s_{1}^{-1}\right)} Q(\sigma)=X(\sigma) t^{-l\left(s^{-1}\right)} .
$$




\section{$\S 7 . \quad$ The Ordinary Case}

In [Kat], Katz defined the ordinarity of a CM-type; the definition is as follows. Take an embedding $\overline{\mathbf{Q}} \hookrightarrow \mathbf{C}_{p}$. Let $L$ be a CM-field and $\Psi$ be a CMtype of $L$. We regard $\Psi$ as a set of isomorphisms of $L$ into $\overline{\mathbf{Q}}$. If the $p$-adic valuations obtained from $\sigma: L \hookrightarrow \mathbf{C}_{p}, \tau: L \hookrightarrow \mathbf{C}_{p}$ are inequivalent whenever $\sigma \in \Psi, \tau \notin \Psi$, we call $\Psi$ ordinary. The following proposition, which seems to be unnoticed before, is easy to prove once formulated.

Proposition 7.1. Let $\left(L^{\prime}, \Psi^{\prime}\right)$ be the reflex of $(L, \Psi)$. Let $\mathcal{P}^{\prime}$ be the prime ideal of $L^{\prime}$ induced by the embedding $L^{\prime} \hookrightarrow \overline{\mathbf{Q}} \hookrightarrow \mathbf{C}_{p}$. Then $\Psi$ is ordinary if and only if $\mathcal{P}^{\prime}$ is unramified over $\mathbf{Q}$ and the degree of $\mathcal{P}^{\prime}$ over $\mathbf{Q}$ is 1 ; $\Psi$ is ordinary for every embedding $\overline{\mathbf{Q}} \hookrightarrow \mathbf{C}_{p}$ if and only if $p$ splits completely in $L^{\prime}$.

Proof. Let $M$ be a CM-field which contains $L$ and is normal over $\mathbf{Q}$. We put

$$
G=\operatorname{Gal}(M / \mathbf{Q}), \quad H=\operatorname{Gal}(M / L) .
$$

Regarding $\Psi$ as a subset of $G / H$, let $S$ be the full inverse image of $\Psi$ under the canonical map $G \longrightarrow G / H$. We put

$$
S^{\prime}=\left\{\sigma^{-1} \mid \sigma \in S\right\}, \quad H^{\prime}=\left\{g \mid g \in G, S^{\prime} g=S^{\prime}\right\} .
$$

By the definition of reflex, we have $\operatorname{Gal}\left(M / L^{\prime}\right)=H^{\prime}$ and $\Psi^{\prime}$ consists of the restrictions of elements of $S^{\prime}$ to $L^{\prime}$.

Let $\widetilde{\mathcal{P}}$ be the prime ideal of $M$ which is induced by the embedding $M \hookrightarrow$ $\overline{\mathbf{Q}} \hookrightarrow \mathbf{C}_{p}$ and let $Z$ be the decomposition group of $\widetilde{\mathcal{P}}$. The mapping

$$
H \backslash G / Z \longrightarrow \sigma(\widetilde{\mathcal{P}}) \cap \mathcal{O}_{L}
$$

gives a bijection between $H \backslash G / Z$ and the prime ideals of $L$ lying over $p$. For an embedding $\sigma: L \hookrightarrow \overline{\mathbf{Q}}$, let $\widetilde{\sigma}$ denote its extension to $M \hookrightarrow \overline{\mathbf{Q}}$. We can regard $\widetilde{\sigma}$ as an element of $G$. Since the prime ideal of $L$ induced by $\sigma$ is $\widetilde{\sigma}^{-1}(\widetilde{\mathcal{P}}) \cap \mathcal{O}_{L}$, we see that $\Psi$ is ordinary if and only if $H \widetilde{\sigma}^{-1} Z \neq H \widetilde{\tau}^{-1} Z$ whenever $\sigma \in \Psi$, $\tau \notin \Psi$. This condition can be written as

$$
S^{\prime} Z \cap S^{\prime} \rho Z=\emptyset
$$

where $\rho$ denotes the complex conjugation. Since $S^{\prime} \sqcup S^{\prime} \rho=G,(7.1)$ is equivalent to $S^{\prime} Z=S^{\prime}$, i.e., $Z \subset H^{\prime}$. The last condition is equivalent to that $\mathcal{P}^{\prime}$ is unramified over $\mathbf{Q}$ and the degree of $\mathcal{P}^{\prime}$ over $\mathbf{Q}$ is 1 . This proves the first assertion. 
The condition that $\Psi$ is ordinary for every embedding $\overline{\mathbf{Q}} \hookrightarrow \mathbf{C}_{p}$ amounts to that (7.1) holds with $g Z g^{-1}$ in place of $Z$ for every $g \in G$. This is equivalent to $g Z g^{-1} \subset H^{\prime}$ and to that $p$ splits completely in $L^{\prime}$. The proof is now complete.

Remark. In view of this proposition, we see that the ordinarity condition of Katz corresponds to the assumption $e=f=1$ in Corollary 1 to Lemma 6.1. The reason is as follows. Let $A$ be an abelian variety of dimension $n$ of CM-type $(L, \Psi)$. Let $\left(L^{\prime}, \Psi^{\prime}\right)$ be the reflex of $(L, \Psi)$. We assume that $A$ and the complex multiplication by $L$ are defined over an algebraic number field $k$. We have $k \supset L^{\prime}$. Then $H^{1}(A)$ determines a motive over $k$ with coefficients in $L$. For a Hecke character $\psi$ of $k$ of the form

$$
\psi((\alpha))=\operatorname{det} \Psi^{\prime}\left(N_{k / L^{\prime}}(\alpha)\right), \quad \alpha \equiv 1 \quad \bmod { }^{\times} \mathfrak{q},
$$

we have $H^{1}(A) \cong M(\psi)$. Here $\operatorname{det} \Psi^{\prime}(x)=\prod_{\sigma \in \Psi^{\prime}} \sigma(x), x \in L^{\prime}$ and $\mathfrak{q}$ is an ideal of $k$ (cf. [S2], Theorem 19.11). For simplicity, assume $k=L^{\prime}$. Notice that $L^{\prime}$ plays the role of $K$ here, in our notation of $\S 2$. Then Proposition 7.1 says that $\Psi$ is ordinary if and only if $e=f=1$ for the prime ideal $\mathfrak{P}\left(=\mathcal{P}^{\prime}\right)$ of $K$.

Now we assume that $\mathfrak{P}$ is degree 1 and unramified over $\mathbf{Q}$, i. e., $e=f=$ 1. Since the restriction of the Frobenius map $\varphi$ to $\overline{\mathbf{Q}}$ is contained in $H=$ $\operatorname{Gal}(\overline{\mathbf{Q}} / K)$, we have $l\left(s^{-1}\right)=l\left(s_{1}^{-1}\right)$. Hence (6.7) yields

$$
\Phi_{\text {cris }}\left(X\left(\sigma_{1}\right)\right) p^{-l\left(s^{-1}\right)} Q(\sigma)=X(\sigma) .
$$

Here $s_{1}$ and $\sigma_{1}$ are defined below (6.6). By (4.6), we have $Q(\sigma)=\sigma(\chi(\mathfrak{P}))$. Hence, by Corollary 1 to Lemma 6.1 , we have $v\left(p^{-l\left(s^{-1}\right)} Q(\sigma)\right)=0$. By Lemma 6.3 , there exists $Y(\sigma) \in \widehat{\mathbf{Q}_{p}^{\text {ur }}}$ such that

$$
\varphi(Y(\sigma)) p^{-l\left(s^{-1}\right)} Q(\sigma)=Y(\sigma)
$$

Then (7.2) can be written as

$$
\Phi_{\text {cris }}\left(X\left(\sigma_{1}\right) Y(\sigma)^{-1}\right)=X(\sigma) Y(\sigma)^{-1} .
$$

Proposition 7.2. Assume that $\sigma_{1}=\sigma$. Then, with $Y(\sigma) \in \widehat{\mathbf{Q}_{p}^{u r}}$ which satisfies (7.3) and $c \in \mathbf{Q}_{p}^{\times}$, we have $(P(\chi))(\sigma)=c Y(\sigma) t^{-l\left(s^{-1}\right)}$.

Proof. Note that $v_{\mathrm{D} R}\left(X(\sigma) Y(\sigma)^{-1}\right)=0$. Then by (7.4) and by [Fo2], Théorème 5.3.7, (iii), we conclude that $X(\sigma)$ differs from $Y(\sigma)$ by the multiplication of an element in $\mathbf{Q}_{p}^{\times}$. 
Remark. The assumption $\sigma_{1}=\sigma$ is satisfied if $p$ splits completely in $E$. In the case $E=E_{0}$, this condition is satisfied if $p$ splits completely in $K$.

Remark. The referee communicated us a more conceptual proof of our results in Sections 6 and 7. Particularly noteworthy is the following result which holds also in the non-ordinary case. When $Q(\chi)$ and $l$ are given, $P(\chi)$ is characterized up to the multiplication of an element in $\left(E \otimes_{\mathbf{Q}} \mathbf{Q}_{p}\right)^{\times}$by the following two conditions.

$$
\begin{gathered}
\left(\operatorname{id}_{E} \otimes \Phi_{\text {cris }}\right)(P(\chi)) \cdot Q(\chi)=P(\chi), \\
P(\chi)(\sigma) \in \mathrm{Fil}^{-l\left(s^{-1}\right)} B_{\mathrm{DR}}, \quad P(\chi)(\sigma) \neq 0,
\end{gathered}
$$

where $\sigma \in J_{E, p}, s \in \operatorname{Gal}(\overline{\mathbf{Q}} / \mathbf{Q}), s \mid E=\sigma$.

\section{$\S 8 . \quad$ Complements}

In this section, we will supply more supporting evidences to Conjecture Q. Let $K$ be a CM-field and $\chi$ be a Grössencharacter of $K$ of conductor $\mathfrak{f}$ which satisfies (2.1). Let $M=M(\chi)$ be the motive attached to $\chi$. For $\tau \in J_{E}$, we define a Grössencharacter $\tau(\chi)$ by

$$
(\tau(\chi))(\mathfrak{a})=\tau(\chi(\mathfrak{a}))
$$

where $\mathfrak{a}$ is a fractional ideal relatively prime to $\mathfrak{f}$. Let $\tau(M)=M(\tau(\chi))$ be the motive attached to $\tau(\chi) ; \tau(M)$ is a motive over $K$ with coefficients in $\tau(E)$. For realizations, we have

$$
H_{*}(\tau(M))=H_{*}(M),
$$

where $*$ stands for Betti, $p$-adic, de Rham and crystalline. In this identification, the $\tau(E)$-module structure on $H_{*}(\tau(M))$ is given by

$$
\tau(e) x=e x, \quad e \in E, x \in H_{*}(M) .
$$

As in $\S 4$, we fix an embedding $\overline{\mathbf{Q}} \subset \overline{\mathbf{Q}_{p}}$ and define the prime ideal $\mathfrak{P}$ of $K$ and the periods $P(\chi)$ and $P(\tau(\chi))$ using this embedding. If $P(\chi)=\sum_{i} a_{i} \otimes b_{i} \in\left(E \otimes_{\mathbf{Q}}\right.$ $\left.B_{\mathrm{DR}}\right)^{\times}$, then $P(\tau(\chi))$ is given by $P(\tau(\chi))=\sum_{i} \tau\left(a_{i}\right) \otimes b_{i} \in\left(\tau(E) \otimes_{\mathbf{Q}} B_{\mathrm{DR}}\right)^{\times}$. For $\sigma \in J_{p, E}$, we have

$$
P(\chi)(\sigma)=\sum_{i} \sigma\left(a_{i}\right) b_{i}
$$


We can identify $J_{E}$ with $J_{p, E}$. For $\sigma \in J_{p, E}$, we have $\sigma \tau^{-1} \in J_{p, \tau(E)}$. Since

$$
P(\tau(\chi))\left(\sigma \tau^{-1}\right)=\sum_{i}\left(\left(\sigma \tau^{-1}\right)\left(\tau\left(a_{i}\right)\right)\right) b_{i}
$$

we obtain

$$
P(\tau(\chi))\left(\sigma \tau^{-1}\right)=P(\chi)(\sigma), \quad \sigma \in J_{p, E} .
$$

We assume that $\chi$ is unramified at $\mathfrak{P}$. Let $Q^{(i)}(\chi) \in\left(E \otimes_{\mathbf{Q}} K_{\mathfrak{P}, 0}\right)^{\times}$denote the quantity defined by (4.2) using the motive $M(\chi)$. By the same reasoning as above, we have

$$
Q^{(i)}(\tau(\chi))\left(\sigma \tau^{-1}\right)=Q^{(i)}(\chi)(\sigma), \quad \sigma \in J_{p, E} .
$$

Here the $\sigma$-component $Q^{(i)}(\chi)(\sigma)$ is defined as in the beginning of $\S 5$.

Now assume that a CM-field $K$ is an abelian extension of a totally real field $F$. For $\tau \in \operatorname{Gal}(K / F)$, we take a Hecke character $\chi$ of the form

$$
\chi((\alpha))=(\rho \tau(\alpha) / \tau(\alpha))^{l}, \quad \alpha \equiv 1 \bmod ^{{ }^{\times}} \mathfrak{f}
$$

with $1 \leqq l \in \mathbf{Z}$. We have $E_{0} \subset K$. Taking a power of $\chi$ if necessary, we assume that $E=K$. Let $V$ denote the $\mathbf{Q}$-subspace of $\mathbf{C}_{p}$ generated by the elements of the form $a \log _{p} b, a \in \mathbf{Q}, b \in K^{\times}$. With this notation, assuming that $\mathfrak{p}$ is unramified in $K$, Conjecture $\mathrm{Q}$ states

$$
l g_{p, K / F}\left(\mathrm{id}, \tau^{-1}\right) \equiv \frac{1}{2 l} \log _{p} Q^{\left(f_{0}\right)}(\chi)(\mathrm{id}) \bmod V .
$$

For $\sigma \in \operatorname{Gal}(K / F)$, apply Conjecture Q to $\sigma(\chi)$ and use (8.2). Then we obtain

$$
l g_{p, K / F}\left(\mathrm{id}, \tau^{-1} \sigma^{-1}\right) \equiv \frac{1}{2 l} \log _{p} Q^{\left(f_{0}\right)}(\chi)(\sigma) \bmod V .
$$

Proposition 8.1. $\quad$ Let $K$ be a CM-field which is abelian over a totally real field $F$. Let $F_{1}$ be an intermediate field between $F$ and $K$. We assume that $F_{1}$ is totally real. Let $\mathfrak{P}$ and $\mathfrak{p}_{1}$ be the prime ideals of $F$ and $F_{1}$ respectively which are obtained by the embeddings $F \subset F_{1} \subset K \subset \mathbf{C}_{p}$. Let $f_{1}$ (resp. $f_{0}$ ) be the degree of $\mathfrak{p}_{1}$ (resp. $\mathfrak{p}$ ) over $\mathbf{Q}$ and put $d=f_{1} / f_{0}$. We assume that $\mathfrak{p}$ is unramified in $K$ and put $\sigma=\left(\frac{K / F}{\mathfrak{p}}\right)$. If Conjecture $Q$ holds, then we have

$$
l g_{p, K / F_{1}}(\mathrm{id}, \tau) \equiv \sum_{i=0}^{d-1} l g_{p, K / F}\left(\mathrm{id}, \tau \sigma^{i}\right) \quad \bmod V, \quad \tau \in \operatorname{Gal}\left(K / F_{1}\right)
$$


Proof. The automorphism $\sigma$ gives a generator of $\operatorname{Gal}\left(K_{\mathfrak{P}} / F_{\mathfrak{p}}\right)$, which can be extended to an element of $\operatorname{Gal}\left(\overline{\mathbf{Q}_{p}} / F_{\mathfrak{P}}\right)$ denoted also by $\sigma$. By (4.5), we have

$$
Q^{\left(f_{1}\right)}(\chi)=\left(\sigma^{d-1} Q^{\left(f_{0}\right)}(\chi)\right) \cdots\left(\sigma Q^{\left(f_{0}\right)}(\chi)\right) Q^{\left(f_{0}\right)}(\chi) .
$$

Take $\tau \in \operatorname{Gal}\left(K / F_{1}\right)$. By (8.3) and (8.4), we obtain

$$
\begin{aligned}
l g_{p, K / F_{1}}\left(\mathrm{id}, \tau^{-1}\right) & \equiv \frac{1}{2 l} \log _{p} Q^{\left(f_{1}\right)}(\chi)(\mathrm{id}) \bmod V, \\
\lg _{p, K / F}\left(\mathrm{id}, \tau^{-1} \sigma^{j}\right) & \equiv \frac{1}{2 l} \log _{p} Q^{\left(f_{0}\right)}(\chi)\left(\sigma^{-j}\right) \bmod V .
\end{aligned}
$$

Since $l g_{p, K / F}\left(\mathrm{id}, \tau^{-1} \sigma^{j}\right) \in F_{\mathfrak{p}}, \sigma$ is continuous and $V$ is stable under $\sigma$, the latter formula implies

$$
\sigma^{j}\left(\log _{p} Q^{\left(f_{0}\right)}(\chi)\left(\sigma^{-j}\right)\right) \equiv \log _{p} Q^{\left(f_{0}\right)}(\chi)\left(\sigma^{-j}\right) \quad \bmod V
$$

We have

$$
\left(\sigma^{j} Q^{\left(f_{0}\right)}(\chi)\right)(\mathrm{id})=\sigma^{j}\left(Q^{\left(f_{0}\right)}(\chi)\left(\sigma^{-j}\right)\right) .
$$

As $\sigma$ is continuous, the asserted formula follows from (8.5).

We are going to prove the relation of Proposition 8.1 in a certain special case. Let $F$ be a totally real algebraic number field. Let $\mathfrak{q}_{0}(F)$ be the product of all prime ideals which divide $p$. We put $\mathfrak{q}(F)=\mathfrak{q}_{0}(F)$ if $p \neq 2, \mathfrak{q}(F)=$ $(2) \mathfrak{q}_{0}(F)$ if $p=2$. Put $\mathfrak{q}=\mathfrak{q}(F)$. Let $\mathfrak{f}$ be an integral ideal of $F$ and $\chi$ be a character of $\mathfrak{C}_{\mathfrak{f}}$. Let $\mathfrak{f}(\chi)$ be the finite part of the conductor of $\chi$. Let $\omega$ be the Teichmüller character of $F$ (cf. I, §2). We regard $\omega \chi$ as a character of $\mathfrak{C}_{\mathfrak{f}(\chi) \mathfrak{q}}$. Let $\left\{C_{j}\right\}_{j \in J}$ be a cone decomposition (cf. I, $\left.\S 1\right)$ and $\left\{\mathfrak{a}_{\mu}\right\}_{\mu=1}^{h_{0}}$ be a complete set of representatives of the narrow ideal classes of F. By (I.2.16) and Theorem I.3.1, we have

$$
L_{p}^{\prime}(0, \omega \chi)=\sum_{\sigma \in J_{F}} \sum_{c \in \mathfrak{C}_{\mathfrak{f}(\chi) \mathfrak{q}}} \chi(c) X_{p}\left(c^{\sigma} ;\left\{C_{j}^{\sigma}\right\}, \mathfrak{a}_{\mu}^{\sigma}\right) .
$$

We assume that $\chi$ is primitive, i.e., $\mathfrak{f}=\mathfrak{f}(\chi)$. We recall the fact that $\chi(\mathfrak{l})=0$ if and only if $\mathfrak{l}$ divides $\mathfrak{f}(\chi)$, where $\mathfrak{l}$ is a prime ideal of $F$. By (I.2.17), we have

$$
L_{p}(0, \omega \chi)=\prod_{\mathfrak{l} \mid \mathfrak{q}}(1-\chi(\mathfrak{l})) L(0, \chi) .
$$

Here $\mathfrak{l}$ extends over all prime ideals of $F$ which divide $\mathfrak{q}$. We put

$$
r_{p}(\chi)=|\{\mathfrak{l}|\mathfrak{q}| \chi(\mathfrak{l})=1\}|,
$$




$$
r_{p}^{*}(\chi)=\operatorname{ord}_{s=0} L_{p}(s, \omega \chi) .
$$

Assume that $\chi$ is totally odd. Then a conjecture of Gross states that $r_{p}^{*}(\chi)=$ $r_{p}(\chi)$. By (8.7), we see that $r_{p}^{*}(\chi)=0$ if and only if $r_{p}(\chi)=0$. We showed that $r_{p}^{*}(\chi) \geqq 2$ if $r_{p}(\chi) \geqq 2$ (cf. Corollary 2 to Theorem I.6.5). Hence $r_{p}(\chi)=1$ if $r_{p}^{*}(\chi)=1$.

Let $V_{F}$ be the $\mathbf{Q}$-subspace of $\mathbf{C}_{p}$ generated by the elements of the form $\log _{p} a, a \in F^{\times}$. For $x, y \in \mathbf{C}_{p}$, we define

$$
x \equiv_{F} y \Longleftrightarrow x-y \in V_{F} .
$$

More generally suppose that $x=x(\chi)$ and $y=y(\chi) \in \mathbf{C}_{p}$ be the quantities which depend on the character $\chi$ of an ideal class group of $F$. Let $\mathbf{Q}(\chi)$ be the field generated by all the values of $\chi$ over $\mathbf{Q}$. We write $x \equiv_{F, \chi} y$ if $x(\chi)-y(\chi)=\sum_{i} t_{i}(\chi) v_{i}$ with $t_{i}(\chi) \in \mathbf{Q}(\chi), v_{i} \in V_{F}$ and if $t_{i}\left(\chi^{\alpha}\right)=t_{i}(\chi)^{\alpha}$ for every $\alpha \in \operatorname{Gal}(\overline{\mathbf{Q}} / \mathbf{Q})$.

Now we assume that $F$ is normal over $\mathbf{Q}$. For $\sigma \in J_{F}$ and a character $\chi$ of $\mathfrak{C}_{\mathfrak{f}(\chi)}$ as above, we define a character $\chi_{\sigma}$ of $\mathfrak{C}_{\mathfrak{f}(\chi)^{\sigma}}$ by

$$
\chi_{\sigma}\left(c^{\sigma}\right)=\chi(c), \quad c \in \mathfrak{C}_{\mathfrak{f}(\chi)} .
$$

We have $\mathfrak{f}\left(\chi_{\sigma}\right)=\mathfrak{f}(\chi)^{\sigma}$. By (8.6), we obtain

$$
L_{p}^{\prime}(0, \omega \chi)=\sum_{\sigma \in J_{F}} \sum_{c \in \mathfrak{C}_{\mathfrak{f}\left(\chi_{\sigma}\right) \mathfrak{q}}} \chi_{\sigma}(c) X_{p}\left(c ;\left\{C_{j}^{\sigma}\right\}, \mathfrak{a}_{\mu}^{\sigma}\right) .
$$

Since $F$ is normal over $\mathbf{Q}$, we have

$$
X_{p}\left(c ;\left\{C_{j}^{\sigma}\right\}, \mathfrak{a}_{\mu}^{\sigma}\right) \equiv_{F} X_{p}\left(c ;\left\{C_{j}\right\}, \mathfrak{a}_{\mu}\right)
$$

by Lemma I.3.3, Cor. to Lemma I.3.4, Cor. to Lemma I.3.5 and (I.3.12). Therefore (8.6) can be written as

$$
L_{p}^{\prime}(0, \omega \chi) \equiv_{F, \chi} \sum_{\sigma \in J_{F}} \sum_{c \in \mathfrak{C}_{\mathfrak{f}\left(\chi_{\sigma}\right) \mathfrak{q}}} \chi_{\sigma}(c) X_{p}\left(c ;\left\{C_{j}\right\}, \mathfrak{a}_{\mu}\right) .
$$

By Lemma I.5.4, Cor. to Lemma I.3.4, Cor. to Lemma I.3.5 and (I.3.12), we have

$$
\begin{gathered}
\sum_{c \in \mathfrak{C}_{\mathfrak{f}\left(\chi_{\sigma}\right) \mathfrak{q}}} \chi_{\sigma}(c) X_{p}\left(c ;\left\{C_{j}\right\}, \mathfrak{a}_{\mu}\right) \\
\equiv_{F, \chi} \prod_{\mathfrak{l} \mid \mathfrak{p}^{-1} \mathfrak{q}_{0}(F)}\left(1-\chi_{\sigma}(\mathfrak{l})\right) \sum_{c \in \mathfrak{C}_{\mathfrak{f}\left(\chi_{\sigma}\right) \mathfrak{p}}} \chi_{\sigma}(c) X_{p}\left(c ;\left\{C_{j}\right\}, \mathfrak{a}_{\mu}\right) .
\end{gathered}
$$


By (8.8), we obtain

$$
L_{p}^{\prime}(0, \omega \chi) \equiv{ }_{F, \chi} \sum_{\sigma \in J_{F}} \prod_{\mathfrak{l} \mid \mathfrak{p}^{-1} \mathfrak{q}_{0}(F)}\left(1-\chi_{\sigma}(\mathfrak{l})\right) \sum_{c \in \mathfrak{C}_{\mathfrak{f}\left(\chi_{\sigma}\right) \mathfrak{p}}} \chi_{\sigma}(c) X_{p}\left(c ;\left\{C_{j}\right\}, \mathfrak{a}_{\mu}\right) .
$$

Now let $F, F_{1}$ and $K$ be as in Proposition 8.1. We put

$$
G=\operatorname{Gal}(K / F), \quad H=\operatorname{Gal}\left(K / F_{1}\right), \quad \mathfrak{q}^{*}=\mathfrak{p}^{-1} \mathfrak{q}_{0}(F), \quad \mathfrak{q}_{1}^{*}=\mathfrak{p}_{1}^{-1} \mathfrak{q}_{0}\left(F_{1}\right)
$$

Take $\psi \in \widehat{H}_{-}$. We have

$$
\operatorname{Ind}_{H}^{G} \psi \cong \oplus_{\chi \in \hat{G}_{-}, \chi \mid H=\psi} \chi
$$

Hence we have

$$
L(s, \psi)=\prod_{\chi \in \hat{G}_{-}, \chi \mid H=\psi} L(s, \chi) .
$$

By the interpolation property of the $p$-adic $L$-function (cf. (I.2.17)), we see that

$$
L_{p}(s, \omega \psi)=\prod_{\chi \in \hat{G}_{-}, \chi \mid H=\psi} L_{p}(s, \omega \chi)
$$

holds in a neighbourhood of $s=0$ (if $p \neq 2$, (8.11) holds for all $s \in \mathbf{Z}_{p}$ ).

To derive the relation of Proposition 8.1 from (8.11), we make the following assumptions.

(I) All of $F, F_{1}$ and $K$ are normal over $\mathbf{Q}$.

(II) For every $\psi \in \widehat{H}_{-}$, we have $r_{p}^{*}(\psi) \leqq 1$.

(III) $\left(\frac{K / F_{1}}{\mathfrak{p}_{1}}\right)$ belongs to the center of $\operatorname{Gal}(K / \mathbf{Q})$.

(IV) $p \neq 2$.

For $i=0,1$, we put

$$
\left(\widehat{H}_{-}\right)_{i}=\left\{\psi \in \widehat{H}_{-} \mid r_{p}^{*}(\psi)=i\right\}, \quad\left(\widehat{G}_{-}\right)_{i}=\left\{\chi \in \widehat{G}_{-} \mid r_{p}^{*}(\chi)=i\right\}
$$

Let $\gamma$ be a conjugacy class of $\operatorname{Gal}(K / \mathbf{Q})$ such that $\gamma \subset \operatorname{Gal}\left(K / F_{1}\right)$. We abbreviate $X_{p}\left(c ;\left\{C_{j}\right\}, \mathfrak{a}_{\mu}\right)$ to $X_{p}(c)$.

First we assume $\psi \in\left(\widehat{H}_{-}\right)_{0}$. By (8.11), we see that $r_{p}^{*}(\chi)=0$ if $\chi \in \widehat{G}_{-}$ satisfies $\chi \mid H=\psi$; we also have

$$
\frac{L_{p}^{\prime}(0, \omega \psi)}{L_{p}(0, \omega \psi)}=\sum_{\chi \in \hat{G}_{-}, \chi \mid H=\psi} \frac{L_{p}^{\prime}(0, \omega \chi)}{L_{p}(0, \omega \chi)}
$$


Applying (8.7) and (8.9) to this equality, we obtain ${ }^{6}$

$$
\begin{gathered}
\sum_{\sigma \in J_{F_{1}}} \frac{1}{L(0, \psi)\left(1-\psi_{\sigma}\left(\mathfrak{p}_{1}\right)\right)} \sum_{c \in \mathfrak{C}_{\mathfrak{f}\left(\psi_{\sigma}\right) \mathfrak{p} 1}} \psi_{\sigma}(c) X_{p}(c) \\
\equiv_{F_{1}, \psi} \sum_{\chi \in \hat{G}_{-}, \chi \mid H=\psi} \sum_{\sigma \in J_{F}} \frac{1}{L(0, \chi)\left(1-\chi_{\sigma}(\mathfrak{p})\right)} \sum_{c \in \mathfrak{C}_{\mathfrak{f}\left(\chi_{\sigma}\right) \mathfrak{p}} \chi_{\sigma}(c) X_{p}(c) .}
\end{gathered}
$$

For $\sigma \in J_{F}$, we have

$$
\frac{1-\psi_{\sigma}\left(\mathfrak{p}_{1}\right)}{1-\chi_{\sigma}(\mathfrak{p})}=\frac{1-\chi_{\sigma}(\mathfrak{p})^{d}}{1-\chi_{\sigma}(\mathfrak{p})}=1+\chi_{\sigma}(\mathfrak{p})+\cdots+\chi_{\sigma}(\mathfrak{p})^{d-1} .
$$

Here we use the same letter $\sigma$ to its extension to $J_{F_{1}}$. By the assumption (III), $\psi_{\sigma}\left(\mathfrak{p}_{1}\right)$ does not depend on $\sigma \in J_{F_{1}}$. Therefore, from (8.12), we have

$$
\begin{aligned}
& \sum_{\sigma \in J_{F_{1}}} \frac{1}{L(0, \psi)} \sum_{c \in \mathfrak{C}_{\mathfrak{f}\left(\psi_{\sigma}\right) \mathfrak{p}_{1}}} \psi_{\sigma}(c) X_{p}(c) \\
\equiv_{F_{1}, \psi} & \sum_{\chi \in \hat{G}_{-}, \chi \mid H=\psi} \sum_{\sigma \in J_{F}} \frac{1+\chi_{\sigma}(\mathfrak{p})+\cdots+\chi_{\sigma}(\mathfrak{p})^{d-1}}{L(0, \chi)} \sum_{c \in \mathfrak{C}_{\mathfrak{f}\left(\chi_{\sigma}\right) \mathfrak{p}}} \chi_{\sigma}(c) X_{p}(c) .
\end{aligned}
$$

Now multiply both sides of (8.13) by $\sum_{\tau \in \gamma} \psi(\tau)$ and take the sum over $\psi \in$ $\left(\widehat{H}_{-}\right)_{0}$. Note that $L\left(0, \psi_{\sigma}\right)=L(0, \psi), \sum_{\tau \in \gamma} \psi_{\sigma}(\tau)=\sum_{\tau \in \gamma} \psi(\tau)$. From the left-hand side we get

$$
\left|J_{F_{1}}\right| \sum_{\psi \in\left(\hat{H}_{-}\right)_{0}} \frac{\sum_{\tau \in \gamma} \psi(\tau)}{L(0, \psi)} \sum_{c \in \mathfrak{C}_{f(\psi) \mathfrak{p}_{1}}} \psi(c) X_{p}(c) .
$$

From the right-hand side, we get

$$
\left|J_{F}\right| \sum_{\chi \in\left(\hat{G}_{-}\right)_{0}} \frac{\left(\sum_{\tau \in \gamma} \chi(\tau)\right)\left(1+\chi(\mathfrak{p})+\cdots+\chi(\mathfrak{p})^{d-1}\right)}{L(0, \chi)} \sum_{c \in \mathfrak{C}_{\mathfrak{f}(\chi) \mathfrak{p}}} \chi(c) X_{p}(c) .
$$

Therefore we obtain

$$
\begin{aligned}
& \frac{1}{|H|} \sum_{\psi \in\left(\hat{H}_{-}\right)_{0}} \frac{\sum_{\tau \in \gamma} \psi(\tau)}{L(0, \psi)} \sum_{c \in \mathfrak{C}_{\mathfrak{f}(\psi) \mathfrak{p} 1}} \psi(c) X_{p}(c) \\
& \equiv_{F_{1}} \frac{1}{|G|} \sum_{\chi \in\left(\hat{G}_{-}\right)_{0}^{\prime}} \frac{\left(\sum_{\tau \in \gamma} \chi(\tau)\right)\left(\sum_{i=0}^{d-1} \chi(\mathfrak{p})^{i}\right)}{L(0, \chi)} \sum_{c \in \mathfrak{C}_{\mathfrak{f}(\chi) \mathfrak{p}}} \chi(c) X_{p}(c) .
\end{aligned}
$$

${ }^{6} \operatorname{In}(8.12)$ and (8.13), we regard the quantities depending on $\psi$ are 0 , if $\psi \notin\left(\widehat{H}_{-}\right)_{0}$. 
Here $\left(\widehat{G}_{-}\right)_{0}^{\prime}$ denotes the subset of $\left(\widehat{G}_{-}\right)_{0}$ consisting of $\chi$ such that $\chi \mid H \in\left(\widehat{H}_{-}\right)_{0}$.

Next let us consider the case where $\psi \in\left(\widehat{H}_{-}\right)_{1}$. Put

$$
\operatorname{Ind}_{H}^{G} \psi=\oplus_{i=1}^{m} \chi_{i} .
$$

By (8.11), we have

$$
L_{p}(s, \omega \psi)=\prod_{i=1}^{m} L_{p}\left(s, \omega \chi_{i}\right) .
$$

Hence there exists an $i_{0}, 1 \leqq i_{0} \leqq m$ such that $r_{p}^{*}\left(\chi_{i_{0}}\right)=1, r_{p}^{*}\left(\chi_{i}\right)=0$ for $i \neq i_{0}$. We may assume that

$$
r_{p}^{*}\left(\chi_{1}\right)=1, \quad r_{p}^{*}\left(\chi_{i}\right)=0, \quad i \geqq 2 .
$$

We put $\chi=\chi_{1}$. Differentiating (8.15) at $s=0$, we obtain

$$
L_{p}^{\prime}(0, \omega \psi)=L_{p}^{\prime}(0, \omega \chi) \prod_{i=2}^{m} L_{p}\left(0, \omega \chi_{i}\right) .
$$

Applying (8.9) to this equation, we obtain ${ }^{7}$

$$
\begin{gathered}
\sum_{\sigma \in J_{F_{1}}} \prod_{\mathfrak{l}_{1} \mid \mathfrak{q}_{1}^{*}}\left(1-\psi_{\sigma}\left(\mathfrak{l}_{1}\right)\right) \sum_{c \in \mathfrak{C}_{\mathfrak{f}\left(\psi_{\sigma}\right) \mathfrak{p}_{1}}} \psi_{\sigma}(c) X_{p}(c) \\
\equiv_{F_{1}, \psi}\left(\prod_{i=2}^{m} L_{p}\left(0, \omega \chi_{i}\right)\right) \sum_{\sigma \in J_{F}} \prod_{\mathfrak{l} \mid \mathfrak{q}^{*}}\left(1-\chi_{\sigma}(\mathfrak{l})\right) \sum_{c \in \mathfrak{C}_{\mathfrak{f}\left(\chi_{\sigma}\right) \mathfrak{p}}} \chi_{\sigma}(c) X_{p}(c) .
\end{gathered}
$$

By the assumptions (I) and (III), we have $\psi\left(\mathfrak{p}_{1}\right)=\psi\left(\mathfrak{p}_{1}^{\sigma}\right)$ for every $\sigma \in J_{F_{1}}$. By the assumption (II), we see that $\psi\left(\mathfrak{p}_{1}\right)=1$ and that the prime factor of $p$ in $F_{1}$ is unique. Hence the prime factor of $p$ in $F$ is also unique. Now dividing both sides by $L(0, \psi)=\prod_{i=1}^{m} L\left(0, \chi_{i}\right)$ and noting the relation (8.7), we obtain

$$
\begin{gathered}
\sum_{\sigma \in J_{F_{1}}} \frac{1}{L(0, \psi)} \sum_{c \in \mathfrak{C}_{\mathfrak{f}\left(\psi_{\sigma}\right) \mathfrak{p} 1}} \psi_{\sigma}(c) X_{p}(c) \\
\equiv_{F_{1}, \psi} \sum_{\sigma \in J_{F}} \frac{1}{L(0, \chi)} \prod_{i=2}^{m}\left(1-\chi_{i}(\mathfrak{p})\right) \sum_{c \in \mathfrak{C}_{\mathfrak{f}\left(\chi_{\sigma}\right) \mathfrak{p}}} \chi_{\sigma}(c) X_{p}(c) .
\end{gathered}
$$

Since

$$
1-\psi\left(\mathfrak{p}_{1}\right) N\left(\mathfrak{p}_{1}\right)^{-s}=\prod_{i=1}^{m}\left(1-\chi_{i}(\mathfrak{p}) N(\mathfrak{p})^{-s}\right),
$$

\footnotetext{
${ }^{7}$ In two formulas below and in (8.17), we regard the quantities depending on $\psi$ are 0 , if $\psi \notin\left(\widehat{H}_{-}\right)_{1}$.
} 
we have

$$
\prod_{i=2}^{m}\left(1-\chi_{i}(\mathfrak{p})\right)=\lim _{s \rightarrow 0} \frac{1-\psi\left(\mathfrak{p}_{1}\right) N\left(\mathfrak{p}_{1}\right)^{-s}}{1-\chi(\mathfrak{p}) N(\mathfrak{p})^{-s}}=\lim _{s \rightarrow 0} \frac{1-N\left(\mathfrak{p}_{1}\right)^{-s}}{1-N(\mathfrak{p})^{-s}}=d .
$$

Therefore we obtain

$$
\begin{array}{r}
\sum_{\sigma \in J_{F_{1}}} \frac{1}{L(0, \psi)} \sum_{c \in \mathfrak{C}_{\mathfrak{f}\left(\psi_{\sigma}\right) \mathfrak{p}_{1}}} \psi_{\sigma}(c) X_{p}(c) \\
\equiv_{F_{1}, \psi} \sum_{\sigma \in J_{F}} \frac{d}{L(0, \chi)} \sum_{c \in \mathfrak{C}_{\mathfrak{f}\left(\chi_{\sigma}\right) \mathfrak{p}}} \chi_{\sigma}(c) X_{p}(c) .
\end{array}
$$

This formula is similar to (8.12). In the above, we defined a mapping

$$
\left(\widehat{H}_{-}\right)_{1} \ni \psi \longrightarrow \chi \in\left(\widehat{G}_{-}\right)_{1} \text {. }
$$

It is easy to see that this mapping is bijective. Now multiplying both sides of (8.17) by $\sum_{\tau \in \gamma} \psi(\tau)$ and taking the sum over $\psi \in\left(\widehat{H}_{-}\right)_{1}$, we obtain

$$
\begin{aligned}
\frac{1}{|H|} \sum_{\psi \in\left(\hat{H}_{-}\right)_{1}} \frac{\sum_{\tau \in \gamma} \psi(\tau)}{L(0, \psi)} \sum_{c \in \mathfrak{C}_{\mathfrak{f}(\psi) \mathfrak{p} 1}} \psi(c) X_{p}(c) \\
\equiv_{F_{1}} \frac{1}{|G|} \sum_{\chi \in\left(\hat{G}_{-}\right)_{1}} \frac{\left(\sum_{\tau \in \gamma} \chi(\tau)\right)\left(\sum_{i=0}^{d-1} \chi(\mathfrak{p})^{i}\right)}{L(0, \chi)} \sum_{c \in \mathfrak{C}_{\mathfrak{f}(\chi) \mathfrak{p}}} \chi(c) X_{p}(c) .
\end{aligned}
$$

Note that

$$
\sum_{i=0}^{d-1} \chi_{j}(\mathfrak{p})^{i}=0, \quad 2 \leqq j \leqq m
$$

when $\operatorname{Ind}_{H}^{G} \psi=\oplus_{j=1}^{m} \chi_{j}$ with $\chi_{1} \in\left(\widehat{G}_{-}\right)_{1}$. Hence in $(8.14),\left(\widehat{G}_{-}\right)_{0}^{\prime}$ can be replaced by $\left(\widehat{G}_{-}\right)_{0}$. By $(8.14)$ and $(8.18)$, we obtain the following proposition.

Proposition 8.2. Let $F, F_{1}$ and $K$ be the same as in Proposition 8.1. We assume that the conditions (I), (II), (III) and (IV) hold. Let $\gamma$ be a conjugacy class of $\operatorname{Gal}(K / \mathbf{Q})$ which is contained in $\operatorname{Gal}\left(K / F_{1}\right)$. Put $\sigma=\left(\frac{K / F}{\mathfrak{p}}\right)$. Then we have

$$
\sum_{\tau \in \gamma} l g_{p, K / F_{1}}(\mathrm{id}, \tau) \equiv_{F_{1}} \sum_{\tau \in \gamma} \sum_{i=0}^{d-1} l g_{p, K / F}\left(\mathrm{id}, \tau \sigma^{i}\right) .
$$

Corollary. If $\tau \in \operatorname{Gal}\left(K / F_{1}\right)$ belongs to the center of $\operatorname{Gal}(K / \mathbf{Q})$, then we have

$$
\lg _{p, K / F_{1}}(\mathrm{id}, \tau) \equiv_{F_{1}} \sum_{i=0}^{d-1} \lg _{p, K / F}\left(\mathrm{id}, \tau \sigma^{i}\right)
$$


Remark. Taking account of the facts given below (8.7), we can weaken the assumption (II) to that $r_{p}(\psi) \leqq 1$ for every $\psi \in \widehat{H}_{-}$. The proof is essentially the same.

The next proposition, which is much easier to prove, gives another functorial property of our symbol.

Proposition 8.3. $\quad$ Let $K$ be a CM-field which is an abelian extension of a totally real field $F$. Let $K_{0}$ be a CM-field such that $F \subset K_{0} \subset K$. Take $\tau \in \operatorname{Gal}\left(K_{0} / F\right)$ and put $\operatorname{Inf}_{K / K_{0}}(\tau)=\tau_{1}+\cdots+\tau_{m}, m=\left[K: K_{0}\right]$. We define $l g_{p, K / F}\left(\mathrm{id}, \operatorname{Inf}_{K / K_{0}}(\tau)\right)=\sum_{i=1}^{m} l_{p, K / F}\left(\mathrm{id}, \tau_{i}\right)$. Then we have

$$
l g_{p, K_{0} / F}(\mathrm{id}, \tau)=l g_{p, K / F}\left(\mathrm{id}, \operatorname{Inf}_{K / K_{0}}(\tau)\right) \text {. }
$$

Proof. Put

$$
G=\operatorname{Gal}(K / F), \quad G_{0}=\operatorname{Gal}\left(K_{0} / F\right) .
$$

By the definition of the symbol, we have

$$
l g_{p, K / F}\left(\mathrm{id}, \tau_{i}\right)=-\frac{\mu\left(\tau_{i}\right)}{2 h_{K}} \log _{p} \alpha_{0}+\frac{1}{|G|} \sum_{\chi \in \hat{G}_{-}} \frac{\chi\left(\tau_{i}\right)}{L(0, \chi)} \sum_{c \in \mathfrak{C}_{\mathfrak{f}(\chi) \mathfrak{p}}} \chi(c) X_{p}(c) .
$$

If $\chi \mid \operatorname{Gal}\left(K / K_{0}\right)$ is non-trivial, we have $\sum_{i=1}^{m} \chi\left(\tau_{i}\right)=0$. If $\chi \mid \operatorname{Gal}\left(K / K_{0}\right)$ is trivial, we can regard $\chi$ as a character of $G_{0}$ and we have $\sum_{i=1}^{m} \chi\left(\tau_{i}\right)=m \chi(\tau)$. Since $\rho \notin \operatorname{Gal}\left(K / K_{0}\right)$, we have $\sum_{i=1}^{m} \mu\left(\tau_{i}\right)=\mu(\tau)$. Hence the asserted formula follows.

We will show that Conjecture $\mathrm{Q}$ is consistent with this proposition. Let $\psi_{0}$ be a Grössencharacter of $K_{0}$ of conductor $\mathfrak{f}_{0}$ which satisfies

$$
\psi_{0}((\alpha))=\left(\rho \tau^{-1}(\alpha) / \tau^{-1}(\alpha)\right)^{l}, \quad \alpha \equiv 1 \quad \bmod { }^{\times} \mathfrak{f}_{0} .
$$

Then Conjecture Q states

$$
l g_{p, K_{0} / F}(\mathrm{id}, \tau) \equiv \frac{1}{2 l} \log _{p} Q^{\left(f_{0}\right)}\left(\psi_{0}\right)(\mathrm{id}) \bmod V
$$

Here $V$ is the same as in (8.3). Put $\psi=\psi_{0} \circ N_{K / K_{0}}$. Then $\psi$ satisfies

$$
\psi((\alpha))=\prod_{i=1}^{m}\left(\rho \tau_{i}^{-1}(\alpha) / \tau_{i}^{-1}(\alpha)\right)^{l}, \quad \alpha \equiv 1 \quad \bmod { }^{\times} \mathfrak{f}
$$


with an ideal $\mathfrak{f}$ of $K$. In view of (3.6), Conjecture Q asserts

$$
l g_{p, K / F}\left(\mathrm{id}, \operatorname{Inf}_{K / K_{0}}(\tau)\right) \equiv \frac{1}{2 l} \log _{p} Q^{\left(f_{0}\right)}(\psi)(\mathrm{id}) \bmod V .
$$

By (3.7), we have

$$
\frac{1}{2 l} \log _{p} Q^{\left(f_{0}\right)}\left(\psi_{0}\right)(\mathrm{id}) \equiv \frac{1}{2 l} \log _{p} Q^{\left(f_{0}\right)}(\psi)(\mathrm{id}) \quad \bmod V .
$$

Hence Conjecture Q is consistent with Proposition 8.3.

\section{Appendix I}

Let $F$ be a totally real algebraic number field of degree $n$. Let $\mathfrak{f}$ be an integral ideal of $F$ and take a class $c \in \mathfrak{C}_{\mathfrak{f}}$. Let

$$
\mathbf{R}_{+}^{n}=\sqcup_{\epsilon \in E_{F}^{+}} \epsilon\left(\sqcup_{j \in J} C_{j}\right)
$$

be a cone decomposition. Take $\mathfrak{a}_{\mu}$ so that $\mathfrak{a}_{\mu} \mathfrak{f}$ and $c$ belong to the same narrow ideal class. Let $V(c)=V\left(c ;\left\{C_{j}\right\}, \mathfrak{a}_{\mu}\right)$ be the quantity defined by (I.1.6). The purpose of this appendix is to prove:

Theorem. $V(c)$ is of the form $\sum_{i=1}^{n-1} a_{i} \log \epsilon_{i}$ with $a_{i} \in F, \epsilon_{i} \in E_{F}^{+}$.

Proof. If the theorem holds for one choice of $\left\{C_{j}\right\}_{j \in J}$ and $\mathfrak{a}_{\mu}$, it holds for all other choices (cf. (I.3.10), (I.3.11), (I.3.12) and [Y4], Chapter III, Lemma 3.13). More precisely, if $\left\{D_{l}\right\}_{l \in L}$ is a cone decomposition and $\mathfrak{a}_{\mu}^{\prime}$ is an integral ideal such that $\mathfrak{a}_{\mu}^{\prime} \mathfrak{f}$ and $c$ belong to the same narrow ideal class, then we have

(2) $V\left(c ;\left\{C_{j}\right\}_{j \in J}, \mathfrak{a}_{\mu}\right)-V\left(c ;\left\{D_{l}\right\}_{l \in L}, \mathfrak{a}_{\mu}^{\prime}\right)=\sum_{i=1}^{n-1} c_{i} \log \epsilon_{i}, \quad c_{i} \in F, \epsilon_{i} \in E_{F}^{+}$

Hence we may assume that $\mathfrak{a}_{\mu}$ is relatively prime to $\mathfrak{f}$.

We are going to apply (2) when $\mathfrak{a}_{\mu}^{\prime}$ is not an integral ideal. Hence some explanations are called for. Let $J_{F}=\left\{\sigma_{1}, \ldots, \sigma_{n}\right\}$ and put $z^{(i)}=z^{\sigma_{i}}$ for $z \in F$. We choose $\sigma_{1}=$ id. We define zeta functions of cones by

$$
\begin{gathered}
\zeta^{(i)}\left(s, C_{j}, \mathfrak{a}_{\mu}\right)=\sum_{z \in\left(\mathfrak{a}_{\mu} \mathfrak{f}\right)^{-1} \cap C_{j},(z) \mathfrak{a}_{\mu} \mathfrak{f} \in c}\left(z^{(i)}\right)^{-s}, \quad 1 \leqq i \leqq n, \\
\zeta^{(i, k)}\left(s, C_{j}, \mathfrak{a}_{\mu}\right)=\sum_{z \in\left(\mathfrak{a}_{\mu} \mathfrak{f}\right)^{-1} \cap C_{j},(z) \mathfrak{a}_{\mu} \mathfrak{f} \in c}\left(z^{(i)} z^{(k)}\right)^{-s}, \quad 1 \leqq i, k \leqq n .
\end{gathered}
$$


Then $V(c)=V\left(c ;\left\{C_{j}\right\}, \mathfrak{a}_{\mu}\right)$ is equal to (cf. [Y4], Chapter III, (3.29), (3.30))

$$
\begin{gathered}
V(c)=\sum_{j \in J}\left(\frac{2}{n} \sum_{k=2}^{n} v_{1, k}^{(j)}-\frac{1}{n^{2}} \sum_{1 \leqq i, k \leqq n, i \neq k} v_{i, k}^{(j)}\right), \\
v_{i, k}^{(j)}=\left.\frac{d}{d s} \zeta^{(i, k)}\left(s, C_{j}, \mathfrak{a}_{\mu}\right)\right|_{s=0} \\
\quad-\left.\frac{d}{d s} \zeta^{(i)}\left(s, C_{j}, \mathfrak{a}_{\mu}\right)\right|_{s=0}-\left.\frac{d}{d s} \zeta^{(k)}\left(s, C_{j}, \mathfrak{a}_{\mu}\right)\right|_{s=0} .
\end{gathered}
$$

When $\mathfrak{a}_{\mu}$ is not an integral ideal, we define $V(c)=V\left(c ;\left\{C_{j}\right\}, \mathfrak{a}_{\mu}\right)$ by (3), (4). Then (I.3.10), (I.3.11) and (I.3.12) are still valid. Hence (2) remains true. We write $C_{j}=C\left(v_{j 1}, v_{j 2}, \ldots, v_{j r(j)}\right), v_{j i} \in \mathcal{O}_{F}$. For the explicit expression (I.1.6), it is valid if $\mathfrak{a}_{\mu}$ is relatively prime to $\mathfrak{f}$ and if every $v_{j i} \in \mathcal{O}_{F} \cap \mathfrak{a}_{\mu}^{-1}$, because Lemma 3.2 of [Y4], Chapter II holds under that condition.

By the generalized Dirichlet theorem, we can take $\beta \in \mathcal{O}_{F}$ so that (i) $\beta \gg 0$. (ii) $\beta \equiv 1 \bmod \mathfrak{f}$. (iii) $(\beta)$ is a prime ideal of degree 1 which does not divide $\mathfrak{a}_{\mu} \mathfrak{f}$ and any $v_{j i}, j \in J, 1 \leqq i \leqq r(j)$. We put $b=N(\beta)$. Now we consider the quantity

$$
V^{\prime}(c)=V\left(c ;\left\{b C_{j}\right\}_{j \in J}, \mathfrak{a}_{\mu}(\beta)^{-1}\right) .
$$

Here $b C_{j}=C_{j}$ as a cone but we use the expression $b C_{j}=C\left(b v_{j 1}, \ldots, b v_{j r(j)}\right)$. In view of (2), it suffices to show

$$
b V^{\prime}(c)=V(c) .
$$

Let us write the formula (I.1.6) for $V^{\prime}(c)$. (Note that $b v_{j i} \in \mathfrak{a}_{\mu}^{-1}(\beta)$.)

$$
\begin{aligned}
V^{\prime}(c) & =\sum_{j \in J}(-1)^{r(j)} \sum_{l}\left[\frac{1}{n} \sum_{k=2}^{n}\left(C_{l, 1, k}\left(A_{j}\right)+C_{l, k, 1}\left(A_{j}\right)\right)\right. \\
& \left.-\frac{1}{n^{2}} \sum_{1 \leqq i, k \leqq n, i \neq k} C_{l, i, k}\left(A_{j}\right)\right] \times \sum_{z \in R^{\prime}\left(b C_{j}, c\right)} \prod_{m=1}^{r(j)} \frac{B_{l_{m}}\left(x_{m}(z)\right)}{l_{m} !} .
\end{aligned}
$$

Here $l=\left(l_{1}, l_{2}, \ldots, l_{r(j)}\right)$ extends over all $r(j)$-tuples of nonnegative integers such that $l_{1}+l_{2}+\cdots+l_{r(j)}=r(j)$. We note that $C_{l, i, k}\left(b A_{j}\right)=C_{l, i, k}\left(A_{j}\right)$. $R^{\prime}\left(b C_{j}, c\right)$ is $R\left(C_{j}, c\right)$ with $b C_{j}$ in place of $C_{j}$ and $\mathfrak{a}_{\mu}(\beta)^{-1}$ in place of $\mathfrak{a}_{\mu}$. 
Explicitly

$$
\begin{array}{r}
R^{\prime}\left(b C_{j}, c\right)=\left\{z=\sum_{m=1}^{r(j)} x_{m}(z) b v_{j m} \mid x_{m}(z) \in \mathbf{Q}, 0<x_{m}(z) \leqq 1,\right. \\
\left.1 \leqq m \leqq r(j), z \in\left(\mathfrak{a}_{\mu} \mathfrak{f}\right)^{-1}(\beta),(z) \mathfrak{a}_{\mu} \mathfrak{f}(\beta)^{-1} \in c\right\} .
\end{array}
$$

To prove (5), it suffices to show that

$$
b \sum_{z \in R^{\prime}\left(b C_{j}, c\right)} \prod_{m=1}^{r(j)} \frac{B_{l_{m}}\left(x_{m}(z)\right)}{l_{m} !}=\sum_{z \in R\left(C_{j}, c\right)} \prod_{m=1}^{r(j)} \frac{B_{l_{m}}\left(x_{m}(z)\right)}{l_{m} !}
$$

for every $l \neq(1,1, \ldots, 1)$, since $C_{l, i, k}\left(A_{j}\right)=0$ for $l=(1,1, \ldots, 1)$.

Take $z=\sum_{m=1}^{r(j)} x_{m}(z) v_{j m} \in R\left(C_{j}, c\right)$. Since $(\beta)$ is a prime ideal of degree 1 , we have

$$
\mathbf{Z} / b \mathbf{Z} \cong \mathcal{O}_{F} /(\beta) \cong\left(\mathfrak{a}_{\mu} \mathfrak{f}\right)^{-1} /\left(\mathfrak{a}_{\mu} \mathfrak{f}\right)^{-1}(\beta) .
$$

Since $(\beta)$ does not divide $v_{j m}$, we can find $a_{m} \in \mathbf{Z}, 0 \leqq a_{m}<b, 1 \leqq m \leqq r(j)$ so that

$$
z+\sum_{m=1}^{r(j)} a_{m} v_{j m} \in\left(\mathfrak{a}_{\mu} \mathfrak{f}\right)^{-1}(\beta)
$$

Let us verify that $w=z+\sum_{m=1}^{r(j)} a_{m} v_{j m} \in R^{\prime}\left(b C_{j}, c\right)$. For the sake of completeness, let us state the next lemma.

Lemma. Let $\mathfrak{q}$ be an integral ideal of $F$. Let $a$ and $b$ be elements of $F$ such that $a-b \in \mathcal{O}_{F}$. Assume that $(a) \mathfrak{q}$ is relatively prime to $\mathfrak{q}$. Then $(b) \mathfrak{q}$ is relatively prime to $\mathfrak{q}$ and we have $(a) \mathfrak{q} \equiv(b) \mathfrak{q} \bmod ^{\times} \mathfrak{q}$.

Proof. We may assume $\mathfrak{q} \neq(1)$. Let $\mathfrak{p}$ be a prime divisor of $\mathfrak{q}$ and let $\mathfrak{p}^{e(\mathfrak{p})}$ be the exact power of $\mathfrak{p}$ which divides $\mathfrak{q}$. By the assumptions, we have $v_{\mathfrak{p}}(a)=v_{\mathfrak{p}}(b)=-e(\mathfrak{p})<0$. Hence $(b) \mathfrak{q}$ is relatively prime to $\mathfrak{q}$. We also have $v_{\mathfrak{p}}\left(b a^{-1}-1\right)=v_{\mathfrak{p}}\left((b-a) a^{-1}\right) \geqq e(\mathfrak{p})$. Hence $(a) \mathfrak{q} \equiv(b) \mathfrak{q} \bmod ^{\times} \mathfrak{q}$ holds.

To show $w \in R^{\prime}\left(b C_{j}, c\right)$, it suffices to check

$$
(w) \mathfrak{a}_{\mu} \mathfrak{f}(\beta)^{-1} \equiv(z) \mathfrak{a}_{\mu} \mathfrak{f} \quad \bmod ^{\times} \mathfrak{f} .
$$

Since $\beta \equiv 1 \bmod \mathfrak{f}$ and $\mathfrak{a}_{\mu}$ is relatively prime to $\mathfrak{f}$, this is equivalent to

$$
(w) \mathfrak{f} \equiv(z) \mathfrak{f} \bmod ^{\times} \mathfrak{f} .
$$


The relation (11) follows from the Lemma stated above.

Conversely take $w=\sum_{m=1}^{r(j)} y_{m} b v_{j m} \in R^{\prime}\left(b C_{j}, c\right)$. We can write $w=$ $\sum_{m=1}^{r(j)}\left(x_{m}+a_{m}\right) v_{j m}$ with $0<x_{m} \leqq 1, a_{m} \in \mathbf{Z}, 0 \leqq a_{m}<b, 1 \leqq m \leqq r(j)$. Put $z=\sum_{m=1}^{r(j)} x_{m} v_{j m}$ and let us verify that $z \in R\left(C_{j}, c\right)$. To this end, it suffices to check (10) which is equivalent to (11). The required relation also follows from the Lemma.

Now let us prove (8). Since $l \neq(1,1, \ldots, 1)$, there exists an index $h$ such that $l_{h}=0$. When $a_{m} \in \mathbf{Z}, 0 \leqq a_{m}<b, 1 \leqq m \leqq r(l), m \neq h$ are given, the value of $a_{h} \in \mathbf{Z}, 0 \leqq a_{h}<b$ satisfying (9) is uniquely determined. Therefore, by the correspondence $R\left(C_{j}, c\right) \longleftrightarrow R^{\prime}\left(b C_{j}, c\right)$ described above and by taking account of $B_{0}(x)=1$, we have

$$
\begin{aligned}
& \sum_{z \in R^{\prime}\left(b C_{j}, c\right)} \prod_{m=1}^{r(j)} \frac{B_{l_{m}}\left(x_{m}(z)\right)}{l_{m} !} \\
= & \sum_{z \in R\left(C_{j}, c\right)} \prod_{m=1, m \neq h}^{r(j)}\left(\sum_{a_{m}=0}^{b-1} \frac{B_{l_{m}}\left(\left(x_{m}(z)+a_{m}\right) / b\right)}{l_{m} !}\right) .
\end{aligned}
$$

Since

$$
\sum_{a=0}^{b-1} B_{k}\left(\frac{x+a}{b}\right)=\frac{1}{b^{k-1}} B_{k}(x), \quad 0 \leqq k \in \mathbf{Z},
$$

and $\sum_{m \neq h}\left(l_{m}-1\right)=1$, we see that the right-hand side is equal to

$$
\frac{1}{b} \sum_{z \in R\left(C_{j}, c\right)} \prod_{m=1}^{r(l)} \frac{B_{l_{m}}\left(x_{m}(z)\right)}{l_{m} !} .
$$

Hence (8) follows. This completes the proof of our theorem.

\section{Appendix II}

In this appendix, we will formulate a slightly different version of Conjecture I.A. We will prove that both formulations are equivalent.

Let $F$ be a totally real algebraic number field of degree $n$. Let $K$ be an abelian extension of $F$. We assume that $K$ is a CM-field. Put $G=\operatorname{Gal}(K / F)$. For $\tau \in G$, put $\mu(\tau)=1$ if $\tau=$ id, $\mu(\tau)=-1$ if $\tau=\rho$ and $\mu(\tau)=0$ if otherwise. For $\tau \in G$, we define

$$
\begin{aligned}
l g_{p, K}^{(B)}(\mathrm{id}, \tau) & =-\frac{\mu(\tau)}{2} \log _{p} \mathfrak{p} \\
& +\frac{1}{|G|} \sum_{\chi \in \hat{G}_{-}} \frac{\chi(\tau)}{L(0, \chi)} \sum_{c \in \mathfrak{C}_{\mathrm{f}(\chi)}} \chi(c) X_{p}(c) .
\end{aligned}
$$


Compare with the definition (I.4.1) and notice that $\sum_{c \in \mathfrak{C}_{\mathrm{f}(x) \mathfrak{p}}}$ in (I.4.1) is replaced by $\sum_{c \in \mathfrak{C}_{\mathrm{f}(\chi)}}$. The previous symbol $l_{p, K}(\mathrm{id}, \tau)$ defined by (I.4.1) will be denoted by $l g_{p, K}^{(A)}(\mathrm{id}, \tau)$ in this appendix.

Conjecture B. Let $F$ and $K$ be as above. Let $\mathfrak{p}$ and $\mathfrak{P}$ be prime ideals of $F$ and $K$ respectively, which are obtained from the embeddings $F \subset K \subset$ $\overline{\mathbf{Q}} \subset \mathbf{C}_{p}$. Put

$$
\mathfrak{P}^{h_{K}}=(\alpha), \quad \alpha \in \mathcal{O}_{K} .
$$

If $\mathfrak{p}$ splits completely in $K$ and if $\mathfrak{p}$ does not divide $\mathfrak{a}_{\mu}$ for $1 \leqq \mu \leqq h_{0}$, then we have

$$
\begin{aligned}
l g_{p, K}^{(B)}(\mathrm{id}, \tau)= & \frac{1}{2 h_{K}} \log _{p}\left(\alpha^{\tau^{-1} \rho} / \alpha^{\tau^{-1}}\right) \\
& +\left[\lg _{K}\left(\mathrm{id}, \tau ;\left\{C_{j}\right\},\left\{\mathfrak{a}_{\mu}\right\}\right)-l g_{K}\left(\mathrm{id}, \tau ;\left\{C_{j}\right\},\left\{\mathfrak{a}_{\mu} \mathfrak{p}^{-1}\right\}\right)\right]_{p} \\
& +\frac{1}{|G|} \sum_{\chi \in \hat{G}_{-}} \frac{\chi(\tau)}{L(0, \chi)} \sum_{c \in \mathfrak{C}_{\mathfrak{f}(\chi)}} \chi(c) V_{p}\left(c ;\left\{C_{j}\right\}, \mathfrak{a}_{\mu} \mathfrak{p}^{-1}\right) .
\end{aligned}
$$

We are going to transform Conjecture I.A to equivalent statements which will eventually lead to Conjecture B. Let $\chi \in \widehat{G}_{-}$. We put

$$
Y_{p}(\chi)=\frac{L(0, \chi)}{2 h_{K}} \sum_{\sigma \in G} \chi(\sigma) \log _{p}\left(\alpha^{\sigma \rho} / \alpha^{\sigma}\right)
$$

We regard $\chi$ as a character of $\mathfrak{C}_{\mathfrak{f}(\chi)}$. We put $\mathfrak{f}=\mathfrak{f}(\chi)$. Conjecture I. $\mathrm{A}^{\prime}$, which is equivalent to Conjecture I.A gives

$$
\sum_{c \in \mathfrak{C}_{\mathfrak{f p}}} \chi(c) G_{p}\left(c ;\left\{C_{j}\right\},\left\{\mathfrak{a}_{\mu}\right\}\right)=Y_{p}(\chi)+\left[\sum_{c \in \mathfrak{C}_{\mathfrak{f p}}} \chi(c) G\left(c ;\left\{C_{j}\right\},\left\{\mathfrak{a}_{\mu}\right\}\right)\right]_{p},
$$

since $V$ and $W$-terms in $X_{p}\left(\chi_{\mathfrak{p}}\right)$ and $\left[X\left(\chi_{\mathfrak{p}}\right)\right]_{p}$ cancel out. Let $\varphi: \mathfrak{C}_{\mathfrak{f p}} \longrightarrow \mathfrak{C}_{\mathfrak{f}}$ be the canonical map. By [Y4], Chapter III, (5.11) and (3.28), we have

$$
\sum_{d \in \varphi^{-1}(c)} G\left(d ;\left\{C_{j}\right\}, \mathfrak{a}_{\mu}\right)=G\left(c ;\left\{C_{j}\right\}, \mathfrak{a}_{\mu} \mathfrak{p}\right)-G\left(\underline{\mathfrak{p}}^{-1} c ;\left\{C_{j}\right\}, \mathfrak{a}_{\mu}\right)
$$

for $c \in \mathfrak{C}_{\mathfrak{f}}$. Here the index $\mu$ is chosen so that $c$ and $\mathfrak{a}_{\mu} \mathfrak{f} \mathfrak{p}$ belong to the same narrow ideal class and $\underline{p}$ denotes the class of $\mathfrak{p}$ in $\mathfrak{C}_{\mathfrak{f}}$. Taking the sum over $c$ noting that $\chi(\underline{\mathfrak{p}})=1$, we obtain

$$
\begin{aligned}
\sum_{c \in \mathfrak{C}_{\mathfrak{f p}}} \chi(c) & G_{p}\left(c ;\left\{C_{j}\right\}, \mathfrak{a}_{\mu}\right)=Y_{p}(\chi) \\
+ & {\left[\sum_{c \in \mathfrak{C}_{\mathfrak{f}}} \chi(c)\left(G\left(c ;\left\{C_{j}\right\}, \mathfrak{a}_{\mu} \mathfrak{p}\right)-G\left(c ;\left\{C_{j}\right\}, \mathfrak{a}_{\mu}\right)\right)\right]_{p} }
\end{aligned}
$$


from $(2)$. Take $1 \leqq e \in \mathbf{Z}$ so that $\mathfrak{p}^{e}=(\beta), \beta \gg 0, \beta \equiv 1 \bmod \mathfrak{f}$. In addition to $\varphi$, let

$$
\varphi_{1}: \mathfrak{C}_{\mathfrak{f}(\beta)} \longrightarrow \mathfrak{C}_{\mathfrak{f} \mathfrak{p}}, \quad \psi: \mathfrak{C}_{\mathfrak{f}(\beta)} \longrightarrow \mathfrak{C}_{\mathfrak{f}}
$$

be the canonical maps. We have $\psi=\varphi \circ \varphi_{1}$. By Lemma I.3.6, we have

$$
\sum_{d \in \psi^{-1}(c)} G_{p}\left(d ;\left\{C_{j}\right\}, \mathfrak{a}_{\mu}\right)=\sum_{d \in \varphi^{-1}(c)} G_{p}\left(d ;\left\{C_{j}\right\}, \mathfrak{a}_{\mu} \mathfrak{p}^{e-1}\right) .
$$

Hereafter we assume that $\mathfrak{p}$ does not divide $\mathfrak{a}_{\mu}$ for $1 \leqq \mu \leqq h_{0}$. We need the following Lemma.

Lemma. Let $\sigma \in J_{F}$ and define a prime ideal $\mathfrak{p}_{\sigma}$ of $F$ as in the beginning of $\S 3$ of part I. We assume $\mathfrak{p}_{\sigma} \nmid \mathfrak{a}_{\mu} \mathfrak{f}$. Take a positive integer e so that

$$
\mathfrak{p}_{\sigma}^{e}=(\alpha), \quad \alpha \gg 0, \quad \alpha \equiv 1 \quad \bmod \mathfrak{f}
$$

Let $\varphi: \mathfrak{C}_{\mathfrak{f}(\alpha)} \longrightarrow \mathfrak{C}_{\mathfrak{f}}$ be the canonical map. Then, for $c \in \mathfrak{C}_{\mathfrak{f}}$, we have

$$
\sum_{d \in \varphi^{-1}(c)} G_{p}\left(d^{\sigma} ;\left\{C_{j}^{\sigma}\right\}, \mathfrak{a}_{\mu}^{\sigma}\right)=G_{p}\left(c^{\sigma} ;\left\{\left(\alpha C_{j}\right)^{\sigma}\right\}, \mathfrak{a}_{\mu}^{\sigma}\right)+\log _{p} \alpha^{\sigma} \sum_{d \in \varphi^{-1}(c)} \zeta_{F}(0, d) .
$$

Proof. Similarly to (I.3.15), we put

$$
R\left(\alpha C_{j}, c ; \mathfrak{a}_{\mu}\right)_{\sigma}^{\prime}=\left\{z \in R\left(\alpha C_{j}, c ; \mathfrak{a}_{\mu}\right) \mid \mathfrak{p}_{\sigma} \nmid(z)\right\} .
$$

Take $c \in \mathfrak{C}_{\mathrm{f}}$ and put $\varphi^{-1}(c)=\left\{c_{1}, \ldots, c_{l}\right\}$. Then the mapping $z \rightarrow \alpha^{-1} z$ gives a bijection between $R\left(\alpha C_{j}, c ; \mathfrak{a}_{\mu}\right)_{\sigma}^{\prime}$ and $\sqcup_{i=1}^{l} R\left(C_{j}, c_{i} ; \mathfrak{a}_{\mu}\right)$. Therefore we have

$$
\sum_{i=1}^{l} \sum_{z \in R\left(C_{j}, c_{i} ; \mathfrak{a}_{\mu}\right)} L \Gamma_{p, r(j)}\left(z^{\sigma}, v_{j}^{\sigma}\right)=\sum_{z \in R\left(\alpha C_{j}, c ; \mathfrak{a}_{\mu}\right)_{\sigma}^{\prime}} L \Gamma_{p, r(j)}\left(\left(\alpha^{-1} z\right)^{\sigma}, v_{j}^{\sigma}\right) .
$$

We have $\left|z^{\sigma}\right|_{p}=1$ for $z \in R\left(\alpha C_{j}, c ; \mathfrak{a}_{\mu}\right)_{\sigma}^{\prime}$. By (I.2.11), we obtain

$$
\begin{aligned}
& \sum_{i=1}^{l} \sum_{z \in R\left(C_{j}, c_{i} ; \mathfrak{a}_{\mu}\right)} L \Gamma_{p, r(j)}\left(z^{\sigma}, v_{j}^{\sigma}\right) \\
= & \sum_{z \in R\left(\alpha C_{j}, c ; \mathfrak{a}_{\mu}\right)_{\sigma}^{\prime}} L \Gamma_{p, r(j)}\left(z^{\sigma}, \alpha^{\sigma} v_{j}^{\sigma}\right)+\log _{p} \alpha^{\sigma} \cdot \zeta_{p, r(j)}\left(0, \alpha^{\sigma} v_{j}^{\sigma}, z^{\sigma}\right) .
\end{aligned}
$$

By the same reason, we have

$$
\sum_{z \in R\left(\alpha C_{j}, c ; \mathfrak{a}_{\mu}\right)_{\sigma}^{\prime}} \zeta_{p, r(j)}\left(0, \alpha^{\sigma} v_{j}^{\sigma}, z^{\sigma}\right)=\sum_{i=1}^{l} \sum_{z \in R\left(C_{j}, c_{i} ; \mathfrak{a}_{\mu}\right)} \zeta_{p, r(j)}\left(0, v_{j}^{\sigma}, z^{\sigma}\right) .
$$


Since

$$
\sum_{j \in J} \sum_{i=1}^{l} \sum_{z \in R\left(C_{j}, c_{i} ; \mathfrak{a}_{\mu}\right)} \zeta_{p, r(j)}\left(0, v_{j}^{\sigma}, z^{\sigma}\right)=\sum_{i=1}^{l} \zeta_{F}\left(0, c_{i}\right)
$$

by Theorem I.3.2, the assertion follows.

By this Lemma, we obtain

$$
\sum_{d \in \psi^{-1}(c)} G_{p}\left(d ;\left\{C_{j}\right\}, \mathfrak{a}_{\mu}\right)=G_{p}\left(c ;\left\{\beta C_{j}\right\}, \mathfrak{a}_{\mu}\right)+\log _{p} \beta \cdot\left(\zeta_{F}(0, c)-\zeta_{F}\left(\underline{\mathfrak{p}}^{-1} c\right)\right)
$$

Therefore we get

$$
\sum_{c \in \mathfrak{C}_{\mathfrak{f}}} \chi(c) G_{p}\left(c ;\left\{\beta C_{j}\right\}, \mathfrak{a}_{\mu}\right)=\sum_{c \in \mathfrak{C}_{\mathfrak{f} \mathfrak{p}}} \chi(c) G_{p}\left(c ;\left\{C_{j}\right\}, \mathfrak{a}_{\mu} \mathfrak{p}^{e-1}\right)
$$

Applying (3) to the right-hand side, we obtain

$$
\begin{aligned}
\sum_{c \in \mathfrak{C}_{\mathfrak{f}}} \chi(c) & G_{p}\left(c ;\left\{\beta C_{j}\right\}, \mathfrak{a}_{\mu}\right)=Y_{p}(\chi) \\
& +\left[\sum_{c \in \mathfrak{C}_{\mathfrak{f}}} \chi(c)\left(G\left(c ;\left\{C_{j}\right\}, \mathfrak{a}_{\mu} \mathfrak{p}^{e}\right)-G\left(c ;\left\{C_{j}\right\}, \mathfrak{a}_{\mu} \mathfrak{p}^{e-1}\right)\right)\right]_{p}
\end{aligned}
$$

We note that (2), (3) and (4) are equivalent. By [Y4], Chapter III, (3.28), (3.42) and Theorem I.3.2, we have

$$
G\left(c ;\left\{\beta^{-1} C_{j}\right\},(\beta) \mathfrak{a}_{\mu}\right)=G\left(c ;\left\{C_{j}\right\}, \mathfrak{a}_{\mu}\right)+\log \beta \cdot \zeta_{F}(0, c) .
$$

Applying this formula to the right-hand side of (4), we obtain

$$
\begin{aligned}
\sum_{c \in \mathfrak{C}_{\mathfrak{f}}} \chi(c) & G_{p}\left(c ;\left\{\beta C_{j}\right\}, \mathfrak{a}_{\mu}\right)=Y_{p}(\chi) \\
+ & {\left[\sum_{c \in \mathfrak{C}_{\mathfrak{f}}} \chi(c)\left(G\left(c ;\left\{\beta C_{j}\right\}, \mathfrak{a}_{\mu}\right)-G\left(c ;\left\{\beta C_{j}\right\}, \mathfrak{a}_{\mu} \mathfrak{p}^{-1}\right)\right)\right]_{p} }
\end{aligned}
$$

Writing $\beta C_{j}$ as $C_{j}$, we obtain

$$
\begin{aligned}
\sum_{c \in \mathfrak{C}_{\mathfrak{f}}} \chi(c) & G_{p}\left(c ;\left\{C_{j}\right\}, \mathfrak{a}_{\mu}\right)=Y_{p}(\chi) \\
+ & {\left[\sum_{c \in \mathfrak{C}_{\mathfrak{f}}} \chi(c)\left(G\left(c ;\left\{C_{j}\right\}, \mathfrak{a}_{\mu}\right)-G\left(c ;\left\{C_{j}\right\}, \mathfrak{a}_{\mu} \mathfrak{p}^{-1}\right)\right)\right]_{p} }
\end{aligned}
$$


By (5), we have

$$
\begin{aligned}
& l g_{p, K}^{(B)}(\mathrm{id}, \tau)=-\frac{\mu(\tau)}{2} \log _{p} \mathfrak{p}+\frac{1}{|G|} \sum_{\chi \in \hat{G}_{-}} \frac{\chi(\tau)}{2 h_{K}} \sum_{\sigma \in G} \chi(\sigma) \log _{p}\left(\alpha^{\sigma \rho} / \alpha^{\sigma}\right) \\
& +\frac{1}{|G|} \sum_{\chi \in \hat{G}_{-}} \frac{\chi(\tau)}{L(0, \chi)} \sum_{c \in \mathfrak{C}_{\mathfrak{f}(\chi)}} \chi(c)\left(V_{p}\left(c ;\left\{C_{j}\right\}, \mathfrak{a}_{\mu}\right)+W_{p}\left(c ; \mathfrak{a}_{\mu}\right)\right) \\
& +\frac{1}{|G|} \sum_{\chi \in \hat{G}_{-}} \frac{\chi(\tau)}{L(0, \chi)}\left[\sum_{c \in \mathfrak{C}_{\mathfrak{f}(\chi)}} \chi(c)\left(G\left(c ;\left\{C_{j}\right\}, \mathfrak{a}_{\mu}\right)-G\left(c ;\left\{C_{j}\right\}, \mathfrak{a}_{\mu} \mathfrak{p}^{-1}\right)\right)\right]_{p}
\end{aligned}
$$

We can transform this formula to

(6)

$$
\begin{aligned}
& l g_{p, K}^{(B)}(\mathrm{id}, \tau)=-\frac{\mu(\tau)}{2} \log _{p} \mathfrak{p}+\frac{1}{2 h_{K}} \log _{p}\left(\alpha^{\tau^{-1} \rho} / \alpha^{\tau^{-1}}\right) \\
& +\left[l g_{K}\left(\mathrm{id}, \tau ;\left\{C_{j}\right\},\left\{\mathfrak{a}_{\mu}\right\}\right)-l g_{K}\left(\mathrm{id}, \tau ;\left\{C_{j}\right\},\left\{\mathfrak{a}_{\mu} \mathfrak{p}^{-1}\right\}\right)\right]_{p} \\
& +\left[\frac{1}{|G|} \sum_{\chi \in \hat{G}_{-}} \frac{\chi(\tau)}{L(0, \chi)} \sum_{c \in \mathfrak{C}_{\mathfrak{f}(\chi)}} \chi(c) V\left(c ;\left\{C_{j}\right\}, \mathfrak{a}_{\mu} \mathfrak{p}^{-1}\right)\right]_{p} \\
& +\frac{1}{|G|} \sum_{\chi \in \hat{G}_{-}} \frac{\chi(\tau)}{L(0, \chi)} \sum_{c \in \mathfrak{C}_{\mathfrak{f}(\chi)}} \chi(c) W_{p}\left(c ; \mathfrak{a}_{\mu}\right) \\
& -\left[\frac{1}{|G|} \sum_{\chi \in \hat{G}_{-}} \frac{\chi(\tau)}{L(0, \chi)} \sum_{c \in \mathfrak{C}_{\mathfrak{f}(\chi)}} \chi(c)\left(W\left(c ; \mathfrak{a}_{\mu}\right)-W\left(c ; \mathfrak{a}_{\mu} \mathfrak{p}^{-1}\right)\right)\right]_{p} .
\end{aligned}
$$

We consider the last term in (6). For $W\left(c ; \mathfrak{a}_{\mu}\right)\left(\right.$ resp. $\left.W\left(c ; \mathfrak{a}_{\mu} \mathfrak{p}^{-1}\right)\right), c$ and $\mathfrak{a}_{\mu} \mathfrak{f}$ (resp. $\left.\mathfrak{a}_{\mu} \mathfrak{p}^{-1} \mathfrak{f}\right)$ belong to the same narrow ideal class. We find

$$
\begin{aligned}
& {\left[\frac{1}{|G|} \sum_{\chi \in \hat{G}_{-}} \frac{\chi(\tau)}{L(0, \chi)} \sum_{c \in \mathfrak{C}_{\mathfrak{f}(\chi)}} \chi(c)\left(W\left(c ; \mathfrak{a}_{\mu}\right)-W\left(c ; \mathfrak{a}_{\mu} \mathfrak{p}^{-1}\right)\right)\right]_{p} } \\
= & {\left[\frac{1}{|G|} \sum_{\chi \in \hat{G}_{-}} \frac{\chi(\tau)}{L(0, \chi)} \sum_{c \in \mathfrak{C}_{\mathfrak{f}(\chi)}} \chi(c) \log \left(\mathfrak{a}_{\mu} \mathfrak{f}(\chi)\right)\left(\zeta_{F}(0, c)-\zeta_{F}\left(0, \underline{\mathfrak{p}}^{-1} c\right)\right)\right]_{p} } \\
& +\log _{p} \mathfrak{p} \cdot \frac{1}{|G|} \sum_{\chi \in \hat{G}_{-}} \chi(\tau) \\
= & \frac{\mu(\tau)}{2} \log _{p} \mathfrak{p}-\frac{1}{|G|} \sum_{\chi \in \hat{G}_{-}} \frac{\chi(\tau)}{L(0, \chi)} \sum_{c \in \mathfrak{C}_{\mathfrak{f}(\chi)}} \chi(c) W_{p}\left(c ; \mathfrak{a}_{\mu}\right),
\end{aligned}
$$

by the definition of $W_{p}$-term and

$$
\frac{1}{|G|} \sum_{\chi \in \hat{G}_{-}} \chi(\tau)=\frac{\mu(\tau)}{2} .
$$


Thus (6) gives the formula of Conjecture B. We see easily that (4), (5) and (6) are equivalent. Therefore Conjecture B is equivalent to Conjecture I.A.

\section{Appendix III}

The purpose of this Appendix is to prove Lemma I.5.2 which was stated without a proof. Let us recall its statement. We fix embeddings $\overline{\mathbf{Q}} \subset \mathbf{C}$, $\overline{\mathbf{Q}} \subset \mathbf{C}_{p}$. Set

$$
M=\left\{\sum_{i=1}^{m} a_{i} \log b_{i} \mid 1 \leqq m \in \mathbf{Z}, a_{i} \in \overline{\mathbf{Q}}, b_{i} \in \overline{\mathbf{Q}}^{\times}\right\} .
$$

Then $M$ is a $\overline{\mathbf{Q}}$-subspace of $\mathbf{C}$. Here, for $\log b_{i}$, we take any branch of $\log$. Hence $\overline{\mathbf{Q}} \pi \subset M$. Define a $\overline{\mathbf{Q}}$-subspace of $\mathbf{C}_{p}$ by

$$
M_{p}=\left\{\sum_{i=1}^{m} a_{i} \log _{p} b_{i} \mid 1 \leqq m \in \mathbf{Z}, a_{i} \in \overline{\mathbf{Q}}, b_{i} \in \overline{\mathbf{Q}}^{\times}\right\} .
$$

By Lemma I.5.1, we can define a $\overline{\mathbf{Q}}$-linear mapping []$_{p}: M \longrightarrow M_{p}$ by

$$
\left[\sum_{i=1}^{m} a_{i} \log b_{i}\right]_{p}=\sum_{i=1}^{m} a_{i} \log _{p} b_{i}, \quad a_{i} \in \overline{\mathbf{Q}}, b_{i} \in \overline{\mathbf{Q}}^{\times} .
$$

Now Lemma I.5.2 states the following.

Proposition. The kernel of the mapping [ ] $p$ is equal to $\overline{\mathbf{Q}} \pi \oplus \overline{\mathbf{Q}} \log p$.

Proof. It is clear that $\overline{\mathbf{Q}} \pi \oplus \overline{\mathbf{Q}} \log p$ is contained in the kernel. We will show the opposite inclusion. Let ||$_{p}$ be the $p$-adic absolute value of $\mathbf{C}_{p}$ normalized by $|p|_{p}=p^{-1}$. Assume that $\sum_{i=1}^{m} a_{i} \log _{p} b_{i}=0$. Replacing $b_{i}$ by $\zeta_{i} p^{w_{i}} b_{i}$ with a root of unity $\zeta_{i}, w_{i} \in \mathbf{Q}$ if necessary, we may assume that

$$
\left|b_{i}-1\right|_{p}<1, \quad 1 \leqq i \leqq m .
$$

Reordering $b_{i}$ if necessary, we may assume that $\left\{\log _{p} b_{1}, \ldots, \log _{p} b_{l}\right\}$ is a basis of the $\overline{\mathbf{Q}}$-vector space spanned by $\log _{p} b_{i}, 1 \leqq i \leqq m$. If $l=m$, then all $a_{i}$ are 0 and there is nothing to prove. Hence we may assume that $1 \leqq l<m$. Then we have

$$
\log _{p} b_{l+j}=\sum_{k=1}^{l} \alpha_{j k} \log _{p} b_{k}, \quad \alpha_{j k} \in \overline{\mathbf{Q}}, \quad 1 \leqq j \leqq m-l .
$$

Put

$$
c_{i}=a_{i}+\sum_{j=1}^{m-l} \alpha_{j i} a_{l+j}, \quad 1 \leqq i \leqq l
$$


Then we have

$$
c_{i}=0, \quad 1 \leqq i \leqq l .
$$

By a theorem of Brumer $[\mathrm{Br}], \log _{p} b_{1}, \ldots, \log _{p} b_{l}, \log _{p} b_{l+j}$ must be linearly dependent over $\mathbf{Q}$. Hence we have $\alpha_{j k} \in \mathbf{Q}$ in (2).

We can find a positive integer $e$ so that $e \alpha_{j k} \in \mathbf{Z}, 1 \leqq k \leqq l$ and that

$$
\begin{gathered}
\left|b_{i}^{e \alpha_{j k}}-1\right|_{p}<p^{-\frac{1}{p-1}}, \quad 1 \leqq i \leqq l, 1 \leqq j \leqq m-l, 1 \leqq k \leqq l \\
\left|b_{l+j}^{e}-1\right|_{p}<p^{-\frac{1}{p-1}}, \quad 1 \leqq j \leqq m-l
\end{gathered}
$$

Since

$$
\log _{p} b_{l+j}^{e}=\sum_{k=1}^{l} \log _{p} b_{k}^{e \alpha_{j k}}
$$

by (2) and the $p$-adic exponential function $\exp _{p}$ satisfies

$$
\exp _{p}\left(\log _{p} x\right)=x, \quad x \in \mathbf{C}_{p}, \quad|x-1|_{p}<p^{-\frac{1}{p-1}},
$$

we obtain

$$
b_{l+j}^{e}=\prod_{k=1}^{l} b_{k}^{e \alpha_{j k}}
$$

Hence we have

$$
\log b_{l+j} \equiv \sum_{k=1}^{l} \alpha_{j k} \log b_{k} \quad \bmod \overline{\mathbf{Q}} \pi
$$

This implies

$$
\sum_{i=1}^{m} a_{i} \log b_{i} \equiv \sum_{i=1}^{l} c_{i} \log b_{i} \equiv 0 \quad \bmod \overline{\mathbf{Q}} \pi
$$

and completes the proof.

\section{References}

[A] G. W. Anderson, Logarithmic derivatives of Dirichlet $L$-functions and the periods of abelian varieties, Compositio Math. 45 (1982), no. 3, 315-332.

[Bl1] D. Blasius, On the critical values of Hecke $L$-series, Ann. of Math. (2) 124 (1986), no. $1,23-63$.

[Bl2] - A p-adic property of Hodge classes on abelian varieties, in Motives (Seattle, WA, 1991), 293-308, Proc. Sympos. Pure Math. 55, Part 2, Amer. Math. Soc., Providence, RI, 1994.

[BO] P. Berthelot and A. Ogus, F-isocrystals and de Rham cohomology. I, Invent. Math. 72 (1983), no. 2, 159-199. 
[Br] A. Brumer, On the units of algebraic number fields, Mathematika 14 (1967), 121124

[Co] P. Colmez, Périodes des variétés abéliennes à multiplication complexe, Ann. of Math. (2) 138 (1993), no. 3, 625-683.

[D] P. Deligne, Valeurs de fonctions $L$ et périodes d'intégrales, in Automorphic forms, representations and L-functions (Proc. Sympos. Pure Math., Oregon State Univ., Corvallis, Ore., 1977), Part 2, 313-346, Proc. Sympos. Pure Math., 33, Amer. Math. Soc., Providence, RI, 1979.

[DMOS] P. Deligne, J. S. Milne, A. Ogus and K. Shih, Hodge cycles, motives, and Shimura varieties, Lecture Notes in Math., 900, Springer, Berlin, 1982.

[DS] E. de Shalit, On monomial relations between $p$-adic periods, J. Reine Angew. Math. 374 (1987), 193-207.

[Fa] G. Faltings, Crystalline cohomology and p-adic Galois-representations, in Algebraic analysis, geometry, and number theory (Baltimore, MD, 1988), 25-80, Johns Hopkins Univ. Press, Baltimore, MD, 1989.

[Fo1] J.-M. Fontaine, Sur certains types de représentations p-adiques du groupe de Galois d'un corps local; construction d'un anneau de Barsotti-Tate, Ann. of Math. (2) 115 (1982), no. 3, 529-577.

[Fo2] Le corps des périodes p-adiques, Astérisque No. 223 (1994), 59-111.

[Gi] R. Gillard, Relations monomiales entre périodes $p$-adiques, Invent. Math. 93 (1988), no. 2, 355-381.

[Gre] R. Greenberg, Trivial zeros of $p$-adic $L$-functions, in $p$-adic monodromy and the Birch and Swinnerton-Dyer conjecture (Boston, MA, 1991), 149-174, Contemp. Math., 165, Amer. Math. Soc., Providence, RI, 1994.

[GK] B. H. Gross and N. Koblitz, Gauss sums and the $p$-adic $\Gamma$-function, Ann. of Math. (2) 109 (1979), no. 3, 569-581.

[GM] H. Gillet and W. Messing, Cycle classes and Riemann-Roch for crystalline cohomology, Duke Math. J. 55 (1987), no. 3, 501-538.

[Gro] B. H. Gross, $p$-adic $L$-series at $s=0$, J. Fac. Sci. Univ. Tokyo Sect. IA Math. 28 (1981), no. 3, 979-994 (1982).

[H] H. Hida, Hilbert modular forms and Iwasawa theory, Oxford Univ. Press, Oxford, 2006.

[I] K. Iwasawa, Lectures on p-adic L-functions, Ann. of Math. Stud., 74, Princeton Univ. Press, Princeton, NJ, 1972.

[JKS] U. Jannsen, S. Kleiman and J-P. Serre (eds.), Motives, Proc. Sympos. Pure Math. 55, Part 1 and 2, Amer. Math. Soc., Providence, RI, 1994.

[K] T. Kashio, On a p-adic analogue of Shintani's formula, J. Math. Kyoto Univ. 45 (2005), no. 1, 99-128.

[Kat] N. M. Katz, p-adic L-functions for CM fields, Invent. Math. 49 (1978), no. 3, 199297.

[KY1] T. Kashio and H. Yoshida, On the p-adic absolute CM-period symbol, in Algebra and number theory, 359-399, Hindustan Book Agency, Delhi, 2005.

[KY2] On $p$-adic absolute CM-periods I, to appear in Amer. J. Math. 130

[O] A. Ogus, A p-adic analogue of the Chowla-Selberg formula, in $p$-adic analysis (Trento, 1989), 319-341, Lecture Notes in Math., 1454, Springer, Berlin, 1990.

[S1] G. Shimura, Automorphic forms and the periods of abelian varieties, J. Math. Soc. Japan 31 (1979), no. 3, 561-592 (= Collected Papers III, [79a]).

[S2] — Abelian varieties with complex multiplication and modular functions, Princeton Univ. Press, Princeton, NJ, 1998.

[Sc] N. Schappacher, Periods of Hecke characters, Lecture Notes in Math., 1301, Springer, Berlin, 1988.

[Sh] T. Shintani, On values at $s=1$ of certain $L$ functions of totally real algebraic number fields, in Algebraic number theory (Kyoto Internat. Sympos., Res. Inst. Math. Sci., Univ. Kyoto, Kyoto, 1976), 201-212, Japan Soc. Promotion Sci., Tokyo, 1977. 
[T] T. Tsuji, Semi-stable conjecture of Fontaine-Jannsen: a survey, Astérisque No. 279 (2002), 323-370.

[Y1] H. Yoshida, On the zeta functions of Shimura varieties and periods of Hilbert modular forms, Duke Math. J. 75 (1994), no. 1, 121-191.

[Y2] _ On absolute CM-periods, in Automorphic forms, automorphic representations, and arithmetic (Fort Worth, TX, 1996), 221-278, Proc. Sympos. Pure Math. 66, Part 1, Amer. Math. Soc., Providence, RI, 1999.

[Y3] _ On absolute CM-periods. II, Amer. J. Math. 120 (1998), no. 6, 1199-1236.

[Y4] - Absolute CM-periods, Mathematical Surveys and Monographs 106, Amer. Math. Soc., Providence, RI, 2003. 